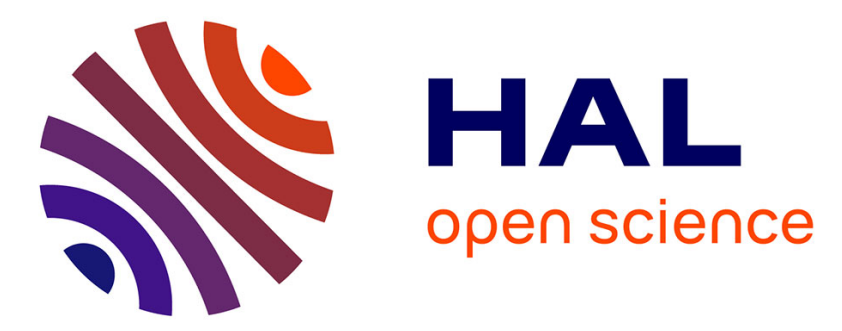

\title{
Phosphorescence excitation mapping and vibrational spectroscopy of HC9N and HC11N cyanopolyynes in organic solvents
}

Urszula Szczepaniak, Kazunori Ozaki, Kaito Tanaka, Yuma Ohnishi, Yoriko Wada, Jean-Claude Guillemin, Claudine Crépin, Robert Kolos, Yusuke Morisawa, Hal Suzuki, et al.

\section{To cite this version:}

Urszula Szczepaniak, Kazunori Ozaki, Kaito Tanaka, Yuma Ohnishi, Yoriko Wada, et al.. Phosphorescence excitation mapping and vibrational spectroscopy of HC9N and HC11N cyanopolyynes in organic solvents. Journal of Molecular Structure, 2020, 1214, pp.128201. 10.1016/j.molstruc.2020.128201 . hal-02563940

\author{
HAL Id: hal-02563940 \\ https://hal.science/hal-02563940
}

Submitted on 6 May 2020

HAL is a multi-disciplinary open access archive for the deposit and dissemination of scientific research documents, whether they are published or not. The documents may come from teaching and research institutions in France or abroad, or from public or private research centers.
L'archive ouverte pluridisciplinaire HAL, est destinée au dépôt et à la diffusion de documents scientifiques de niveau recherche, publiés ou non, émanant des établissements d'enseignement et de recherche français ou étrangers, des laboratoires publics ou privés. 
Credit Author Statement:

Ms. Ref. No.: MOLSTRUC-D-20-00568

Dr. Urszula Szczepaniak: As the leading researcher of this project, she presented initial idea of phosphorescence experiments and started the collaboration by visiting Kindai University.

Mr. Kazunori Ozaki: In the early stage of the phosphorescence experiments in Kindai University, he supported the sample preparation with various polyyne moleules.

Mr. Kaito Tanaka: He worked for preparation of purified solution samples of cyanopolyyne molecules for detailed phosphorescence spectroscopy.

Mr. Yuma Ohnishi: For the IR absorption spectroscopy, he prepared preparative-scale sample solutions of $\mathrm{HC}_{9} \mathrm{~N}$ and $\mathrm{HC}_{11} \mathrm{~N}$.

Dr. Yoriko Wada: Serving as the instructor on sample preparation, she measured IR absorption spectra of $\mathrm{HC}_{9} \mathrm{~N}$ and $\mathrm{HC}_{11} \mathrm{~N}$ with careful treatments of sample concentration and volumes.

Prof. Jean-Claude Guillemin: As an expert of the cyanopolyyne research, he gave the ideas for treatments of the reactive cyanopolyyne molecules.

Prof. Claudine Crépin: As a pioneer of the phosphorescence spectroscopy of matrix isolated cyanopolyyne molecules, comments and discussions are given to the present spectroscopic data.

Prof. Robert Kołos: As an expert of the spectroscopy of matrix isolated cyanopolyynes, discussions are made for the interpretation of spectra, especially phosophorescence.

Prof. Yusuke Morisawa: His expertise on vibrational spectroscopy was highly appreciated upon the discussion of the IR and Raman spectra of cyanopolyyne molecules.

Dr. Hal Suzuki: He worked for the development of the experimental setup of the cryogenic system for phosphorescence spectroscopy.

Prof. Tomonari Wakabayashi: Phosphorescence spectroscopy and interpretation of the spectra were conducted under his leadership. 

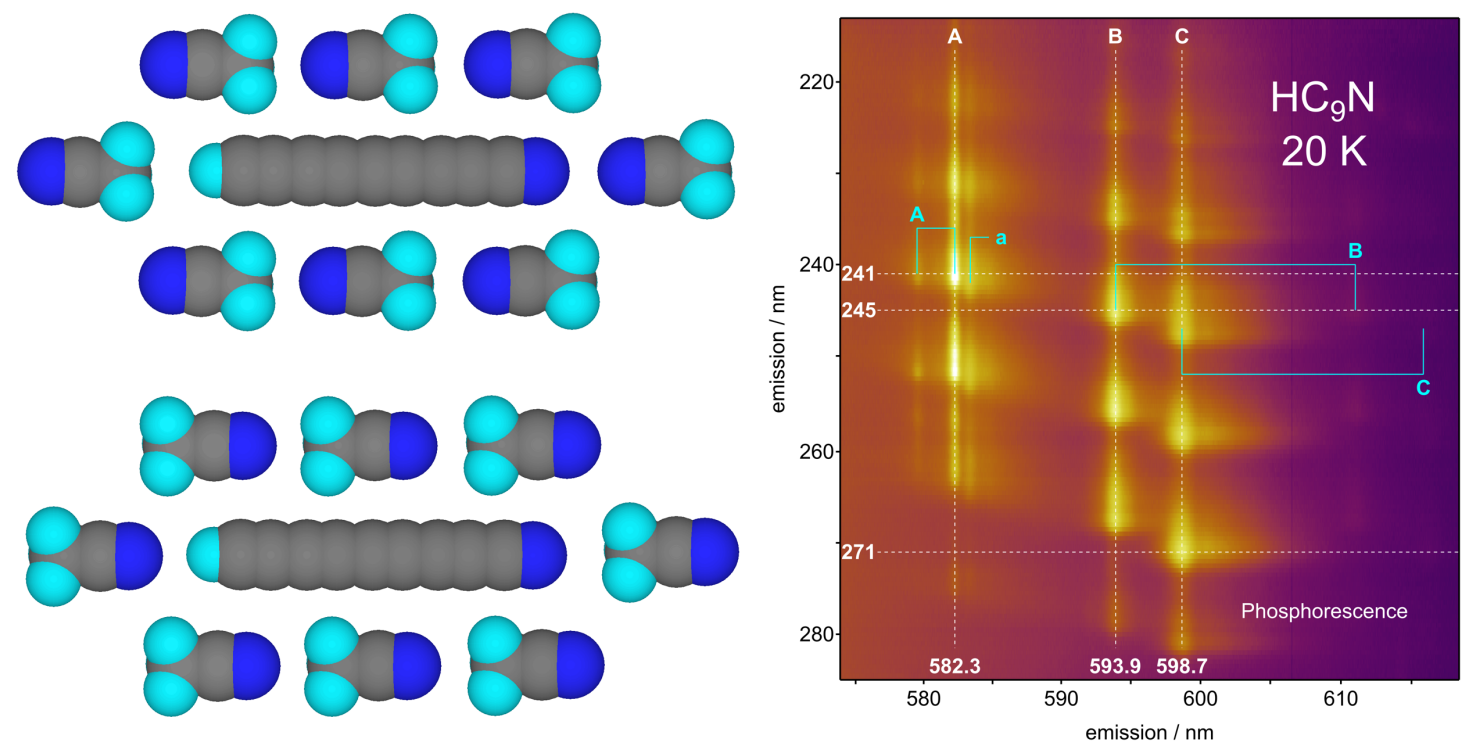
Resubmitted on 2020.4.1 after revision. J. Mol. Struct, Special Issue dedicated to Prof. Zofia Mielke.

\title{
Phosphorescence excitation mapping and vibrational spectroscopy of $\mathrm{HC}_{9} \mathrm{~N}$ and $\mathrm{HC}_{11} \mathrm{~N}$ cyanopolyynes in organic solvents
}

Urszula Szczepaniak, ${ }^{1}$ Kazunori Ozaki, ${ }^{2}$ Kaito Tanaka, ${ }^{2}$ Yuma Ohnishi, ${ }^{2}$ Yoriko Wada, ${ }^{2}$ Jean-Claude Guillemin, ${ }^{3}$ Claudine Crépin, ${ }^{4}$ Robert Kołos, ${ }^{5}$ Yusuke Morisawa, ${ }^{2}$ Hal Suzuki, ${ }^{2}$ and Tomonari Wakabayashi ${ }^{2 *}$

${ }^{1}$ IRsweep, Laubisrütistrasse 44, 8712 Stäfa, Switzerland

${ }^{2}$ Department of Chemistry, Kindai University, Higashi-Osaka 577-8502, Japan

${ }^{3}$ Univ Rennes, Ecole Nationale Supérieure de Chimie de Rennes, CNRS, ISCR - UMR 6226, F-35000 Rennes, France

${ }^{4}$ Université Paris-Saclay, CNRS, Institut des Sciences Moléculaires d'Orsay, 91405, Orsay, France

${ }^{5}$ Institute of Physical Chemistry, Polish Academy of Sciences, Kasprazaka 44/52, Warsaw 01-224, Poland

*Email: wakaba@chem.kindai.ac.jp

\begin{abstract}
Cyanopolyyne molecules, $\mathrm{HC}_{9} \mathrm{~N}$ and $\mathrm{HC}_{11} \mathrm{~N}$, were isolated in solutions and $\mathrm{UV}$, IR, and resonance Raman spectra were measured for the study of their electronic and vibrational properties. Strong signals were observed both in the IR and resonance Raman spectra for the stretching vibrational mode of the sp-hybridized linear carbon chain in the electronic ground state, i.e., $\sigma_{4}$ at $2141 \mathrm{~cm}^{-1}$ for $\mathrm{HC}_{9} \mathrm{~N}$ and $\sigma_{6}$ at $2105 \mathrm{~cm}^{-1}$ for $\mathrm{HC}_{11} \mathrm{~N}$. Trapped in cryogenic solid acetonitrile matrix hosts at $20 \mathrm{~K}$, transitions in phosphorescence, $\tilde{a}^{3} \Sigma^{+} \rightarrow \tilde{X}^{1} \Sigma^{+}$, were observed for $\mathrm{HC}_{9} \mathrm{~N}$ at $582.3 \mathrm{~nm}(0-0)$ and longer wavelengths and for $\mathrm{HC}_{11} \mathrm{~N}$ at $643.7 \mathrm{~nm}(0-0)$ and longer wavelengths. Electronic transitions in the $\mathrm{UV},{ }^{1} \Sigma^{+} \leftarrow \tilde{X}^{1} \Sigma^{+}$, were elucidated by phosphorescence excitation mapping to observe asymmetric patterns with sharp emission-absorption features explainable by Shpolsky effects. For $\mathrm{HC}_{9} \mathrm{~N}$, three distinct trapping sites were discernible in solid acetonitrile, while the phosphorescence spectra were blurred in solid $n$-hexane. The observed phosphorescence lifetime of $\mathrm{HC}_{9} \mathrm{~N}$ was longer than that of
\end{abstract}


$\mathrm{HC}_{11} \mathrm{~N}$, comparable to the trend reported for the series of cyanopolyyne molecules in solid krypton matrix hosts.

\section{Introduction}

Cyanopolyynes, a series of sp-hybridized linear carbon chain molecules terminated by a hydrogen atom at one end and by a cyano-group at the other, $\mathrm{H}(\mathrm{C} \equiv \mathrm{C})_{n} \mathrm{C} \equiv \mathrm{N}$, have been targets of spectroscopic investigations both in space and laboratories [1-12]. Rotationally resolved spectra of microwave emission are widely used for the detection of the series of molecules and are exploited as a probe for molecular evolutions in circumstellar shells of red giants or late AGB stars as well as in molecular clouds [1-5]. Synthetic cyanopolyynes are accessible in the laboratory for gas phase spectroscopy and matrix isolation spectroscopy [6-11]. Photochemistry undergoes in inert cryogenic matrices and is explored to elongate the carbon chain of cyanopolyyne molecules [7-9]. Recently, phosphorescence of cyanopolyynes, $\mathrm{HC}_{5} \mathrm{~N}, \mathrm{HC}_{7} \mathrm{~N}$ and $\mathrm{HC}_{9} \mathrm{~N}$, was detected in solid rare-gas matrices at cryogenic temperature and their vibronic spectra were obtained as action spectra by phosphorescence excitation [9,10,12]. Methylcyanopolyynes are also target molecules for spectroscopy [13-15]. Laser ablation of carbon particles in liquid acetonitrile has opened a way for production of longer polyynes and cyanopolyynes in a milligram order to handle them in organic solvents under ambient temperature [16-20]. Further stabilization is acquired for hydrogen-capped polyyne molecules trapped in solid host environments such as single wall carbon nanotubes (SWNTs) [21-23] and polyvinyl alcohol (PVA) [24].

Increasing interests on carbon species of sp-hybridization are found in the field of nanomaterials as well as astrochemistry. The series of cyanopolyyne molecules, now accessible in the laboratory, are promising model compounds not only for spectroscopy but also for building blocks of novel compounds of sp-hybridized linear carbon chain species. As the interstellar molecules, the presence of $\mathrm{HC}_{9} \mathrm{~N}$ has been confirmed based on microwave emission spectroscopy of molecular rotational transitions $[1,3,4]$, while the detection of $\mathrm{HC}_{11} \mathrm{~N}$ was negated recently by estimating its abundance below an expected amount from the extrapolation [5]. When they are detected in other spectral regions by their electronic and vibrational transitions, the spectral information not only 
reconfirms their abundance in space, especially $\mathrm{HC}_{11} \mathrm{~N}$, but also provides data for the study of photochemical conditions in the interstellar medium.

Matrix isolation spectroscopy combined with theoretical interpretation based on $a b$ initio molecular orbital calculations has been extensively developed as a powerful tool for thorough studies of photochemistry, molecular structures, and intermolecular interactions of reactive species at cryogenic temperature [25-27]. We report here spectroscopic investigations of two cyanopolyynes, namely $\mathrm{HC}_{9} \mathrm{~N}$ and $\mathrm{HC}_{11} \mathrm{~N}$ in solutions at ambient temperature, by UV absorption, infrared (IR) absorption, and resonance Raman spectroscopy. Phosphorescence spectra of $\mathrm{HC}_{9} \mathrm{~N}$ and $\mathrm{HC}_{11} \mathrm{~N}$ are measured in cryogenic matrix hosts of solid acetonitrile or $n$-hexane at $20 \mathrm{~K}$. Phosphorescence excitation mapping is performed for comparison with UV absorption spectra in the solutions to discuss the presence of multiple trapping sites and host-guest interactions in the solid matrices.

\section{Experimental}

\section{Sample preparation and UV absorption spectroscopy}

Preparation and characterization of laser ablated polyyne molecules are described elsewhere [16-20]. Briefly, cyanopolyyne molecules are formed by laser ablation (Continuum Powerlite 8010, $1064 \mathrm{~nm}, 0.5 \mathrm{~J} / \mathrm{pulse}, 10 \mathrm{~Hz}$ ) of graphite particles suspended in acetonitrile (Nacalai Guaranteed Reagent, Acetonitrile, $\geq 99.5 \% \mathrm{CH}_{3} \mathrm{CN}$ ) and separated in size by high performance liquid chromatography (HPLC) [17]. UV absorption spectra are measured for checking purity and for adjusting concentration by using a spectrophotometer (Jasco V-670). Similarly to the hydrogen-capped polyynes [28], cyanopolyynes have molar absorption coefficients of $\sim 10^{5} \mathrm{~L} \mathrm{~mol}^{-1} \mathrm{~cm}^{-1}$. Disregarding vibronic details, interpolated UV absorption coefficients of $\varepsilon \approx 2.2 \times 10^{5}$ and $\approx 2.7 \times 10^{5} \mathrm{~L} \mathrm{~mol}^{-1} \mathrm{~cm}^{-1}$ are applicable to $\mathrm{HC}_{9} \mathrm{~N}$ and $\mathrm{HC}_{11} \mathrm{~N}$, respectively. For spectroscopy in $n$-hexane, cyanopolyyne molecules, e.g., $\mathrm{HC}_{9} \mathrm{~N}$, are extracted from the solution of acetonitrile into $n$-hexane, then residual acetonitrile is removed by HPLC eluted by $n$-hexane (Kishida Chameleon Reagent, Hexane, $>96 \% n$-hexane). 


\section{IR absorption spectroscopy}

For IR absorption spectroscopy [20], a few milligrams of $\mathrm{HC}_{9} \mathrm{~N}$ molecules are extracted into $n$-hexane, concentrated by evaporating $n$-hexane to a volume of $\approx 3 \mathrm{~mL}$, and $\approx 3 \mathrm{~mL}$ of carbon tetrachloride, $\mathrm{CCl}_{4}$, is added then evaporated further to a volume of $\approx 3 \mathrm{~mL}$. Residual $n$-hexane is removed from the crude $\mathrm{CCl}_{4}$ solution of $\mathrm{HC}_{9} \mathrm{~N}$ using HPLC eluted by $\mathrm{CCl}_{4}$ and the resulting fraction of $\mathrm{HC}_{9} \mathrm{~N} / \mathrm{CCl}_{4}$ is concentrated by evaporation to a volume of $\approx 0.5 \mathrm{~mL}$ for the IR measurement.

The 0.1-mm-pathlength IR cell with $\mathrm{KBr}$ windows is filled with the solution of $\mathrm{HC}_{9} \mathrm{~N}$ or $\mathrm{HC}_{11} \mathrm{~N}$ in $\mathrm{CCl}_{4}$ at a concentration of $\sim 10^{-2} \mathrm{~mol} \mathrm{~L}^{-1}$. IR absorption spectra are measured at ambient temperature in a range of $400-4000 \mathrm{~cm}^{-1}$ at a resolution of 1.0 $\mathrm{cm}^{-1}$ using an FTIR spectrometer (Nicolet Magna 750, KBr beam splitter, DTGS detector) [20]. Whole system is covered by a rubber-sealed container box and purged inside with dry nitrogen (Iwatani $\mathrm{N}_{2}$-gas generator $\mathrm{GN}-20,20 \mathrm{~L} / \mathrm{min}$ ) to diminish absorption lines of water vapor and carbon dioxide.

\section{Resonance Raman spectroscopy}

Concentration of $\mathrm{HC}_{9} \mathrm{~N}$ in liquid $n$-hexane is adjusted to the absorbance of $A=0.6-1.2$ at the UV absorption maximum for the optical pathlength of $1.0 \mathrm{~cm}$. A transparent $1.0-\mathrm{cm}$ quartz cell filled with the 4-mL solution in $n$-hexane is placed in a cell holder where the solution is photoexcited by a pulsed UV laser beam of a 4-mm diameter (Sirah Cobra Dye/SHG or VersaScan MB/uvScan OPO/SHG, pumped by 3rd harmonics of INDI-40 Nd:YAG) [18]. The excitation wavelength was tuned at $264 \mathrm{~nm}$ (Coumarin 153/SHG) for $\mathrm{HC}_{9} \mathrm{~N}$ and at $274 \mathrm{~nm}(\mathrm{OPO} / \mathrm{SHG})$ for $\mathrm{HC}_{11} \mathrm{~N}$. The scattered light perpendicular to both directions of propagation and polarization of the linearly polarized laser beam was collected by a quartz lens and routed to the entrance slit of the UV spectrometer (McPherson Model 209, $f=1.33 \mathrm{~m}, 1200 \mathrm{G} / \mathrm{mm}$ blazed at $300 \mathrm{~nm}$ ) equipped with an array detector (PI ICCD $1024 \times 256$ pixels, ST138). The resonance 
Raman scattering spectra are transferred to a PC using WinSpec 3.2 and processed by Igor Pro 8.04.

\section{Phosphorescence Spectroscopy}

The purified solution of $\mathrm{HC}_{9} \mathrm{~N}$ or $\mathrm{HC}_{11} \mathrm{~N}$ is concentrated to a volume of $\approx 1.0 \mathrm{~mL}$ to have a concentration of $\sim 10^{-3} \mathrm{~mol} \mathrm{~L}^{-1}$ and introduced through a 1/16-inch tubing into a vacuum system at $\sim 10^{-5} \mathrm{~Pa}$ of the cryostat (Daikin V202CL). The solution is sprayed then condensed onto the surface of a copper slab cooled at $20 \mathrm{~K}$ in the cryostat to form millimeters-thick solid matrix samples of the mixture, $\mathrm{HC}_{9} \mathrm{~N} / \mathrm{CH}_{3} \mathrm{CN}, \mathrm{HC}_{9} \mathrm{~N} / n$-hexane, or $\mathrm{HC}_{11} \mathrm{~N} / \mathrm{CH}_{3} \mathrm{CN}$. Phosphorescence signals are recorded by three measurement modes: (1) dispersed phosphorescence spectra are recorded at a fixed excitation wavelength using a spectrometer $(\mathrm{PI} 320, f=0.3 \mathrm{~m}, 1200 \mathrm{G} / \mathrm{mm}$ blazed at $500 \mathrm{~nm}$ or $600 \mathrm{G} / \mathrm{mm}$ blazed at $1000 \mathrm{~nm}$ ) equipped with a liquid- $\mathrm{N}_{2}$-cooled array detector (PI PyLoN, OE256), (2) dispersed phosphorescence spectra are recorded redundantly during the excitation-wavelength scan in a range of $213-302 \mathrm{~nm}$ with an increment of $0.5 \mathrm{~nm}$ using a spectrometer (Acton SP300i, $f=0.3 \mathrm{~m}, 1200 \mathrm{G} / \mathrm{mm}$ blazed at $500 \mathrm{~nm}$ or 600 $\mathrm{G} / \mathrm{mm}$ blazed at $500 \mathrm{~nm}$ ) equipped with an array detector (PI SPEC10), and (3) phosphorescence excitation spectra are recorded on a digitizing oscilloscope (LeCroy WavePro 954) by plotting the phosphorescence intensity at a fixed wavelength during the excitation-wavelength scan using a photomultiplier detector (Hamamatsu R928). For the mode (3), a time window, e.g., between 2 and $20 \mathrm{msec}$, is set to avoid instantaneous scattering upon the excitation pulse for collecting only delayed emission to smooth out the relatively featureless background. To suppress the second-order dispersion of the grating spectrometers, colored glass filters (Schott long-pass filters, GG395, GG475, or OG570) are used for cutting the shorter wavelength light. Phosphorescence mapping is made from the series of spectra in the mode (2), while phosphorescence lifetimes are obtained from the decay profile in the mode (3). Commonly for the three modes (1) - (3), excitation is performed by using a tunable pulsed laser system (GWU VersaScan MB/uvScan OPO/SHG pumped by 3rd 
harmonics of INDI-40 Nd:YAG, $7 \mathrm{~nm}$ duration) and the wavelength is calibrated and monitored by using a wavelength meter (GWU LambdaScan).

\section{Results and discussion}

\section{A. UV, IR, and Raman spectra in solutions}

Figure 1 shows UV absorption spectra of $\mathrm{HC}_{9} \mathrm{~N}$ and $\mathrm{HC}_{11} \mathrm{~N}$ in $n$-hexane. The absorption maximum is at $245.6 \mathrm{~nm}$ for the former and at $264.4 \mathrm{~nm}$ for the latter. In addition to the vibrational progression of a stretching mode toward shorter wavelengths, which is similar to the spectra of hydrogen-capped polyynes, $\mathrm{H}(\mathrm{C} \equiv \mathrm{C})_{n} \mathrm{H} \quad[16,18-20,28]$, shoulders and weaker bands are discernible in the longer wavelength region [17]. Transitions to electronic excited states, to which the transition from the ground state is symmetry forbidden for centrosymmetric $\mathrm{H}(\mathrm{C} \equiv \mathrm{C})_{n} \mathrm{H}$ molecules of $D_{\infty \mathrm{h}}$ symmetry, are activated for asymmetric $\mathrm{H}(\mathrm{C} \equiv \mathrm{C})_{n} \mathrm{C} \equiv \mathrm{N}$ molecules of $C_{\infty \mathrm{v}}$ symmetry and observed as a part of the allowed transition, ${ }^{1} \Sigma^{+} \leftarrow \tilde{X}^{1} \Sigma^{+}$. The observed UV absorption features are summarized in Table 1.

Figure 1.

Table 1.

Figure 2 shows IR and resonance Raman spectra for $\mathrm{HC}_{9} \mathrm{~N}$ and $\mathrm{HC}_{11} \mathrm{~N}$. Stretching modes of the sp-carbon chain are peaking in a range of $2028-2239 \mathrm{~cm}^{-1}$ for both molecules. Among others, the strongest peaks at $2141 \mathrm{~cm}^{-1}$ for $\mathrm{HC}_{9} \mathrm{~N}$ and at $2105 \mathrm{~cm}^{-1}$ for $\mathrm{HC}_{11} \mathrm{~N}$ are downshifting with the increasing carbon chain length, while the frequencies of the modes at high and low frequency ends do not depend on the carbon chain length (for vibrational properties by B3LYP/cc-pVQZ molecular orbital calculations, see Table S1 and Figs. S1 and S2 in supplementary data). All the stretching $\sigma$ modes in this frequency region of $2150 \pm 150 \mathrm{~cm}^{-1}$, namely 5 modes, $\sigma_{2}$ $\sigma_{6}$, for $\mathrm{HC}_{9} \mathrm{~N}$ and 6 modes, $\sigma_{2}-\sigma_{7}$, for $\mathrm{HC}_{11} \mathrm{~N}$, are both IR and Raman active for cyanopolyynes of $C_{\infty \mathrm{v}}$ symmetry, though some of them have only negligible intensities, e.g., the IR $\sigma_{4}$ mode of $\mathrm{HC}_{11} \mathrm{~N}$. The highest frequency peak in this region, i.e., $\sigma_{2}$ at 
$2239 \mathrm{~cm}^{-1}$ for $\mathrm{HC}_{9} \mathrm{~N}$ and $\sigma_{2}$ at $2235 \mathrm{~cm}^{-1}$ for $\mathrm{HC}_{11} \mathrm{~N}$, appears with substantial IR absorption intensity relative to the main peak, while the corresponding Raman mode signal is relatively weak. In this collective stretching mode of the $-(C \equiv C)_{n}-C \equiv N$ chain, the bond length of the polarized $\mathrm{C} \equiv \mathrm{N}$ bonding changes relatively largely compared to the other stretching modes at lower frequencies. Conversely, the lack of these bands in the measured Raman spectra is coherent with their expected weakness (for calculated intensities, see Table S1 and Ref. 12). For both $\mathrm{HC}_{9} \mathrm{~N}$ and $\mathrm{HC}_{11} \mathrm{~N}$, strong IR signals were observed for the highest frequency $\mathrm{CH}$ stretching $\sigma_{1}$ mode at $3302-3303 \mathrm{~cm}^{-1}$ and for the low frequency $\mathrm{CH}$ bending $\pi$ mode at $626-532 \mathrm{~cm}^{-1}$ (not shown in Fig. 2). The observed IR and Raman peaks are compiled in Table 2.

Figure 2.

Table 2.

For $\mathrm{HC}_{9} \mathrm{~N}$, a weak IR absorption at $2174 \mathrm{~cm}^{-1}$ is an order of magnitude stronger for the predicted intensity for $\sigma_{3}$ and assigned to an overtone of the pseudosymmetric $\sigma_{8}$ stretching mode [12]. For $\mathrm{HC}_{11} \mathrm{~N}$, a group of IR absorption lines including the strongest signal at $2105 \mathrm{~cm}^{-1}$ and those in its vicinity are attributable to $\sigma_{6}$, which is strongly perturbed by overtones and/or combinations. The nearby $\sigma_{5}$ mode is possibly perturbed also.

Note here that, for the resonance Raman spectroscopy, the excitation wavelength was tuned to a maximum or an edge of a weaker UV absorption band at longer wavelength to avoid serious self-absorption of Stokes lines. During the measurement of the Raman spectra, decay of the Raman signals was observed for the target molecule and some additional signals appear, e.g., a peak with an asterisk in the Raman spectrum of $\mathrm{HC}_{9} \mathrm{~N}$ in Fig. 2, indicating decomposition of $\mathrm{HC}_{9} \mathrm{~N}$ molecules in $n$-hexane upon absorption of the UV photons for the excitation.

\section{B. Phosphorescence of $\mathrm{HC}_{9} \mathrm{~N}$}


Figure 3 plots dispersed phosphorescence spectra of $\mathrm{HC}_{9} \mathrm{~N}$ molecules trapped in solid acetonitrile at $20 \mathrm{~K}$. Typical spectra are shown in Fig. 3a for which the excitation wavelength is set at $250.0,255.0$, or $271.0 \mathrm{~nm}$. Four bands in the electronic transition, $\tilde{a}^{3} \Sigma^{+} \rightarrow \tilde{X}^{1} \Sigma^{+}$, are observed upon the 250.0 -nm excitation with maxima at 582.3, 665.4, 775.6, and $927.8 \mathrm{~nm}$ for $0-0,0-1,0-2$, and 0-3 transitions, respectively, comprising vibrational progression of the $\sigma_{4}$ mode of $\mathrm{HC}_{9} \mathrm{~N}$ for collective stretching motions of the carbon chain. Increments between adjacent pairs of bands are 2146, 2135, and 2115 $\mathrm{cm}^{-1}$, in excellent agreement with the 1-0 fundamental for the $\sigma_{4}$ mode in the electronic ground state as observed at $2141 \mathrm{~cm}^{-1}$ in the IR and Raman spectra in Fig. 2 (see also Table 2). The major bands in the dispersed phosphorescence spectra of $\mathrm{HC}_{9} \mathrm{~N}$ in solid acetonitrile are listed in Table 3.

Figure 3.

Table 3.

The three spectra in Fig. 3a show peaks at the same wavelengths but with different intensities upon the different wavelength excitations. They are grouped into three series of progressions, namely A, B, and C. In Fig. 3b, the top trace in Fig. 3a is reproduced as a function of wavenumber, $\mathrm{cm}^{-1}$, after subtraction of a featureless background. An intense peak in each $0-\mathrm{v}$ band $(\mathrm{v}=0-4)$, indicated as $\mathrm{A}$, is accompanied by broader peaks at lower energies, indicated as B and C. The separation of the peak B from the peak $A$ increases as $335,346,356$, and $370 \mathrm{~cm}^{-1}$ as the vibrational progression of the $\sigma_{4}$ mode increases from $\mathrm{v}=0$ to $\mathrm{v}=1,2$, and 3 . The same trend is confirmed for the peak C. This trend is not compatible with the anharmonicity behavior of a vibrational mode. Moreover, relative intensity of the three peaks, A, B, and C, drastically changes for the different excitation energies as shown in Fig. 3a. These observations indicate that $\mathrm{HC}_{9} \mathrm{~N}$ molecules in solid acetonitrile are accommodated in three types of trapping sites where spatial arrangement of surrounding $\mathrm{CH}_{3} \mathrm{CN}$ molecules are distinctly different for Site A, Site B and Site C. The phosphorescence peaks for Site A are as sharp as those reported in a solid Ar or $\mathrm{Kr}$ matrix [12].

To see the difference of the spectral features, A, B, and C, in more detail, Fig. 4 illustrates phosphorescence excitation mapping for the $0-0$ band of $\mathrm{HC}_{9} \mathrm{~N}$ in solid 
acetonitrile at $20 \mathrm{~K}$. The two-dimensional (2D) contour plot in colors depicts emission intensity as functions of the emission and excitation wavelengths. Horizontal cross sections provide dispersed phosphorescence spectra as shown atop the 2D-map for selected excitation wavelengths of 241,245 , and $271 \mathrm{~nm}$, for which the emission intensities at 582.3, 593.9, and $598.7 \mathrm{~nm}$ are the maximum for the peaks $\mathrm{A}, \mathrm{B}$, and $\mathrm{C}$, respectively. Vertical cross sections constitute phosphorescence excitation spectra as shown to the right of the 2D-map for selected emission wavelengths for the relevant peaks $\mathrm{A}, \mathrm{B}$, and $\mathrm{C}$. In the $2 \mathrm{D}$-map, four major spots are vertically aligned along each line of the emission wavelengths at $582.3 \mathrm{~nm}$ for A, $593.9 \mathrm{~nm}$ for B, and $598.7 \mathrm{~nm}$ for C. This 4-band pattern shifts down on the map (red-shifts) when the detection wavelength changes in the direction from $\mathrm{A}$ to $\mathrm{C}$. The mapping pattern clearly shows one-to-one correspondence between an emission peak and an excitation profile and is rationalized again by the presence of three distinct types of trapping sites for $\mathrm{HC}_{9} \mathrm{~N}$ molecules in solid acetonitrile.

\section{Figure 4.}

Note here that the excitation at $271 \mathrm{~nm}$ gives rise to the phosphorescence emission almost uniquely from Site C. This selective excitation is possible when the $\mathrm{HC}_{9} \mathrm{~N}$ molecule in a specific Site $\mathrm{C}$ has substantial absorption efficiency for the low energy UV photon relative to the molecule in the other Site A or B. The above mentioned red-shifts both in the excitation and emission energies indicate that energy gaps between the electronic ground state and the singlet/triplet excited states get smaller from A to C. A larger gap or a larger transition energy for A indicates the preferential stabilization of the ground-state $\mathrm{HC}_{9} \mathrm{~N}$ molecules in Site A. Conversely, the $\mathrm{HC}_{9} \mathrm{~N}$ molecules in Site B and $\mathrm{C}$ are relatively destabilized for the ground state.

Table 4 compiles minor features observed in Figs. 3 and 4, namely satellite peaks and shoulders in the phosphorescence of $\mathrm{HC}_{9} \mathrm{~N}$ in solid acetonitrile. In Fig. 3a, a satellite peak at $583.4 \mathrm{~nm}$ next to the main peak of Site A at $582.3 \mathrm{~nm}$ is as sharp as the main peak and red-shifted by $\approx 30 \mathrm{~cm}^{-1}$ from the main peak. The intensity of the satellite peak relative to the main peak increases from $\mathrm{v}=0$ to $\mathrm{v}=1$ and 2 for $0-\mathrm{v}$ bands in the $\sigma_{4}$ progression. Moreover, in the mapping in Fig. 4, the maximum excitation wavelength 
for the satellite peak "a" is downshifted by $\approx 1 \mathrm{~nm}\left(\approx 170 \mathrm{~cm}^{-1}\right)$ from that for the main peak "A". Furthermore, the appearance of this satellite peak resembles to the appearance of the secondary peak in the literature, which is well resolved in the solid Ar and $\mathrm{Kr}$ matrix hosts and assigned to $\mathrm{HC}_{9} \mathrm{~N}$ molecules trapped in a secondary matrix site [12]. Another satellite locating at higher energy is separated by $78-97 \mathrm{~cm}^{-1}$ from the main peak $\mathrm{A}$ for $0-\mathrm{v}$ bands $(\mathrm{v}=0-2)$ in the $\sigma_{4}$ progression. Having the same maximum excitation wavelength with the main peak $A$, this satellite is associated with a modification of the trapping site A. For peaks with such minute energy differences as less than $\approx 100 \mathrm{~cm}^{-1}$, presence of an isotopolog, $\mathrm{H}^{13} \mathrm{C}^{12} \mathrm{C}_{8} \mathrm{~N}$ (a total $\approx 9 \%$, i.e., $\approx 1 \%$ for each of the nine isotopomers), and corresponding zero-point-energy differences may also be subjects for considerations [29-31].

Table 4.

Concerning weak bands at $611.4 \mathrm{~nm}\left(1.635 \times 10^{4} \mathrm{~cm}^{-1}\right)$ and $617.6 \mathrm{~nm}\left(1.619 \times 10^{4} \mathrm{~cm}^{-1}\right)$ in Fig. 3a, dim spots in the mapping in Fig. 4 are relevant. The former shares the excitation maximum at $245 \mathrm{~nm}$ for $\mathrm{B}$, while the latter shares the excitation maximum at $247 \mathrm{~nm}$ for C. Difference frequencies from the corresponding major bands are $482 \mathrm{~cm}^{-1}$ for $\mathrm{B}$ and $510 \mathrm{~cm}^{-1}$ for $\mathrm{C}$, which are assignable to the $\pi_{4}$-bending fundamental [12].

Figure 5 shows scanned excitation spectra of $\mathrm{HC}_{9} \mathrm{~N}$, obtained with the measurement mode (3), by motoring the emission intensity at $582.3 \mathrm{~nm}$ for $\mathrm{A}, 593.9 \mathrm{~nm}$ for B, and $598.7 \mathrm{~nm}$ for C. The strongest band peaking at $240.7 \mathrm{~nm}$ for A shifts to the red at 244.9 $\mathrm{nm}$ for B. The other bands for B are shifted also to longer wavelengths compared to those for A. UV absorption spectrum of the solution of $\mathrm{HC}_{9} \mathrm{~N}$ in acetonitrile is shown for comparison (dashed line). Dotted lines are the excitation spectra obtained with the measurement mode (2) as reproduced from the right panel in Fig. 4 with vertical scaling. Peak positions and band widths are fitting well with the spectra between the mode (2) and (3), except for the spectral features in the dotted line spectrum for C, i.e., peaks at 247, 258, and $271 \mathrm{~nm}$. In Site $\mathrm{C}$, the trapping environment for the $\mathrm{HC}_{9} \mathrm{~N}$ molecule can be modified to get stronger absorption efficiency for these excitation wavelengths, which might be developed under the long-term repetitive excitation cycle for the phosphorescence-mapping measurement. 
Figure 5.

Figure 6a shows phosphorescence excitation mapping and a typical dispersed phosphorescence spectrum of $\mathrm{HC}_{9} \mathrm{~N}$ in solid $n$-hexane at $20 \mathrm{~K}$. Within a relatively wide spectral range of $560-820 \mathrm{~nm}$, three phosphorescence bands of the vibrational $\sigma_{4}$ progression are covered in one frame. The major bands in the dispersed phosphorescence spectra of $\mathrm{HC}_{9} \mathrm{~N}$ in $n$-hexane are listed in Table 3. The $2 \mathrm{D}$ contour plot shows some asymmetric red spots along each of the vertical lines for the $0-0,0-1$, or 0-2 bands. Each spot is elongated from up-left to down-right. In contrast to the spots in acetonitrile in Fig. 4, the phosphorescence features in $n$-hexane are not clearly resolved to be classified as distinct matrix sites. Nevertheless, as plotted in Fig. 6b, phosphorescence excitation spectra exhibit spectral features corresponding to absorption bands in the solution of $n$-hexane (dashed line in blue). In Table 1, observed bands in the phosphorescence excitation spectra of $\mathrm{HC}_{9} \mathrm{~N}$ in solid $n$-hexane in Fig. 6 as well as those in solid acetonitrile in Fig. 5 are listed for comparison with absorption features in solutions and with phosphorescence excitation peaks observed in the solid krypton matrix host [12].

Figure 6.

A difference to be noted here is that the spectral feature at $\approx 255 \mathrm{~nm}$ is not clearly discernible for the UV absorption in liquid acetonitrile and for the phosphorescence excitation in solid acetonitrile (Site A). However, peak positions as a whole by the phosphorescence excitation of $\mathrm{HC}_{9} \mathrm{~N}$ in solid $n$-hexane are not much different from those in solid acetonitrile (dotted line) and UV absorption bands in liquid acetonitrile (dashed line in purple) [17], bearing resemblance to a better resolved and consequently more complicated spectrum measured in solid krypton [12]. Phosphorescence excitation spectra are more complex than absorption spectra. It is because, at low temperatures, each of the single vibronic bands gets narrower and becomes well resolved, in contrast to blurred bands overlapping in a solution. In addition, the presence of multiple trapping sites makes the spectrum more complex in solid matrix hosts, whereas spectral details are obscured in solutions by averaging over a variety of instantaneous orientations of surrounding molecules. 
Comparing the phosphorescence excitation with the UV absorption in more detail, the low energy part is more intense in excitation than in absorption for both acetonitrile in Fig. 5 and $n$-hexane in Fig. 6b. For the spectral region of $200-285$ nm, multiple transitions are predicted from the ground state, $\tilde{X}^{1} \Sigma^{+}$, to several electronic excited states, $\widetilde{D}^{1} \Delta, \tilde{E}^{1} \Sigma^{+}, \tilde{F}^{1} \Sigma^{-}, \tilde{G}^{1} \Delta, \widetilde{H}^{1} \Sigma^{+}[12]$. Among them, transitions to the ${ }^{1} \Sigma^{+}$states, $\tilde{E}$ ( $f=$ 2.8) and $\widetilde{H}(f=1.7)$, are fully allowed by the electric dipole mechanism, while transitions to the other ${ }^{1} \Sigma^{-}$and ${ }^{1} \Delta$ states are forbidden but getting allowed via Herzberg-Teller coupling [32-34] with activation or deactivation of a $\pi$-symmetry vibration [12]. For symmetry forbidden singlet-singlet transitions of centrosymmetric polyyne molecules, $\mathrm{H}(\mathrm{C} \equiv \mathrm{C})_{n} \mathrm{H}$, the mechanism of intensity borrowing and the type of inducing modes are discussed in some details [35]. In the cryogenic solid matrix hosts where the surrounding solvent molecules are tightly packed and their orientations are fixed, the electrostatic field in a trapping site for the target molecule can be anisotropic, which may break the symmetry selection rule. Accordingly, vibronic transitions via Herzberg-Teller coupling are promoted to be observed more intense than in solutions.

The structure in excitation shown in Figs. 4 and 5 for acetonitrile and in Fig. $6 \mathrm{~b}$ for $n$-hexane has some similarities with the excitation spectrum in Fig. 7 in Ref. 12 for solid krypton. The low energy part shows a vibronic structure different from that of the high energy part, in agreement with the assignment to multiple excited states [12]. In particular, the spectral feature at $255 \mathrm{~nm}$ observed in $n$-hexane both for UV absorption (shoulder) and phosphorescence excitation (peak) could correspond to the band at 252.5 $\mathrm{nm}$ observed in solid $\mathrm{Kr}$ [12], which was difficult to include in a vibronic progression.

\section{Phosphorescence of $\mathrm{HC}_{11} \mathrm{~N}$}

Figure 7 shows a typical phosphorescence spectrum of $\mathrm{HC}_{11} \mathrm{~N}$ in solid acetonitrile at 20 $\mathrm{K}$. With the excitation at $267.0 \mathrm{~nm}$ (solid line), three peaks at 643.7, 745.8, and 881.7 $\mathrm{nm}$ are conspicuous for vibronic 0-0, 0-1, and 0-2 transitions, respectively, in the $\tilde{a}^{3} \Sigma^{+}$ $\rightarrow \tilde{X}^{1} \Sigma^{+}$system. These peaks are very weak at the excitation of $281.0 \mathrm{~nm}$ (dotted line), while the broad bands toward the low energy side are observed relatively strongly. The vibrational progression is due to excitation of the stretching $\sigma_{6}$ mode of $\mathrm{HC}_{11} \mathrm{~N}$. 
Increment between the $0-0$ and $0-1$ bands, $2108 \mathrm{~cm}^{-1}$, is in very good agreement with the strong IR and Raman mode signal of $\sigma_{6}$ at $2105 \mathrm{~cm}^{-1}$ in Fig. 2. The narrow lines and the relevant broad bands are both belonging to $\mathrm{HC}_{11} \mathrm{~N}$ and indicative of different trapping sites. The peaks in the dispersed phosphorescence spectra of $\mathrm{HC}_{11} \mathrm{~N}$ in solid acetonitrile are listed in Table 3.

Figure 7.

By monitoring the phosphorescence intensity at $644 \mathrm{~nm}(0-0)$, the excitation wavelength is scanned continuously to obtain the excitation spectrum in Fig. 8. The action spectrum (solid line) of this measurement mode (3) is in good agreement with the absorption spectrum in the solution (dotted line), showing relatively narrow band shapes near the peak maxima. The bands in the phosphorescence excitation spectrum of $\mathrm{HC}_{11} \mathrm{~N}$ in solid acetonitrile are listed in Table 1 for comparison with the UV absorption bands.

Figure 8.

For more details, a series of emission spectra of an expanded portion around the phosphorescence $0-0$ band at $644 \mathrm{~nm}$ are recorded repeatedly during the scan of the excitation wavelength in a range of $213-302 \mathrm{~nm}$ by the mode (2) and plotted in Fig. 9 (left panel). From top to bottom, the characteristic cycle is observed several times, with a broad band that appears at shorter wavelength, shifts to longer wavelength, and fades. The observation is rationalized by the idea that a $\mathrm{HC}_{11} \mathrm{~N}$ molecule absorbing a high (low) energy UV photon emits a high (low) energy visible photon as phosphorescence. This trend is seen more clearly in the phosphorescence excitation mapping in Fig. 9 (right panel), i.e., several bumps tailing from up-left to down-right, each of which corresponds to the UV absorption band (c.f. dotted line in Fig. 8).

Figure 9.

The most significant feature of the phosphorescence mapping in Fig. 9 is the narrow peak at $644 \mathrm{~nm}$ on top of the broad band, which does not shift by the excitation wavelength. The upper state of the emission, $\tilde{a}^{3} \Sigma^{+}$, as well as the lower state, $\tilde{X}^{1} \Sigma^{+}$, is populated as a well-defined state in the matrix host. This phenomenon is explainable by 
Shpolsky effects [36], where the host molecules are crystallized around the guest molecule, providing a unique matrix environment of a single trapping site. When the action spectrum of the excitation is deduced only from the areal magnitude of the narrow peak at $644 \mathrm{~nm}$, for which the contribution from the broad band below the peak is subtracted, the spectrum shows narrow peaks also in the excitation spectrum as in Fig. 10. Major high energy bands exhibit relatively narrow peaks, although two low energy bands are relatively broad. However, increments between adjacent peaks from low energy are 1724, 1723, 1881, and $1812 \mathrm{~cm}^{-1}$, being irregular and even increasing in energy. This is not compatible with the idea for assigning them to a single vibrational mode progression in a single electronic potential surface. Moreover, it is apparent from Table 1 that the absorption bands of $\mathrm{HC}_{11} \mathrm{~N}$ readily show the same irregularity. This is because of still unresolved vibronic features even under the condition of cryogenic matrix experiments. As is demonstrated in the excitation spectrum of matrix isolated $\mathrm{HC}_{9} \mathrm{~N}$ molecules in cryogenic solid krypton [12], the excitation spectra as well as the absorption spectra are constituted intrinsically by more complex vibronic features due to the effects by strong intensity borrowing between many electronic states.

Figure 10.

\section{Phosphorescence lifetimes}

Phosphorescence lifetimes were measured by temporal decay profiles of the emission intensity. Several bands at particular excitation and emission wavelengths were examined to realize that the decay constant is more or less the same for the same molecule, indicating that the observed lifetime reflects the population decay of the lowest triplet state, $\tilde{a}^{3} \Sigma^{+}$. The observed lifetimes at $20 \mathrm{~K}$ with fitting error bars are 11.2 $\pm 0.1 \mathrm{~ms}$ in acetonitrile and $9.7 \pm 0.1 \mathrm{~ms}$ in $n$-hexane for $\mathrm{HC}_{9} \mathrm{~N}$ and $7.6 \pm 0.1 \mathrm{~ms}$ in acetonitrile for $\mathrm{HC}_{11} \mathrm{~N}$. For $\mathrm{HC}_{9} \mathrm{~N}$, the slightly shorter lifetime in $n$-hexane than that in acetonitrile may be attributable to the larger number of vibrational degrees of freedom for the host $n$-hexane molecules, leading to enhanced energy dissipation. Although several milliseconds deviations are admitted from different settings for the termination resistance in the measurement system, a clear trend is confirmed that the longer 
cyanopolyyne, $\mathrm{HC}_{11} \mathrm{~N}$, has a shorter lifetime than $\mathrm{HC}_{9} \mathrm{~N}$. This is in good agreement with the trend for reported lifetimes of $40 \mathrm{~ms}$ for $\mathrm{HC}_{5} \mathrm{~N}, 8.2 \mathrm{~ms}$ for $\mathrm{HC}_{7} \mathrm{~N}$ and $3.9 \mathrm{~ms}$ for $\mathrm{HC}_{9} \mathrm{~N}$ in solid $\mathrm{Kr}$ matrices [10,12].

\section{E. Transition energies and the molecular size}

Figure 11 summarizes the experimental transition wavelengths of the phosphorescence 0-0 band for cyanopolyynes $\mathrm{HC}_{2 n+1} \mathrm{~N}(n=2-5)$ as a function of the size of the conjugated linear carbon chain, i.e., the number of carbon atoms in a molecule. The UV absorption maxima of cyanopolyynes $\mathrm{HC}_{2 n+1} \mathrm{~N}(n=3-6)$ and hydrogen-capped polyynes $\mathrm{C}_{2 n} \mathrm{H}_{2}(n=3-7)$ are plotted for reference. Both types of transitions in the visible and UV regions show a systematic trend proportional to the molecular size, as expected for the particle-in-a-box model. The upper state of the phosphorescence, $\tilde{a}^{3} \Sigma^{+} \rightarrow \tilde{X}^{1} \Sigma^{+}$, stems from the same electron configuration as the upper state of the lowest-energy fully-dipole-allowed transition, ${ }^{1} \Sigma^{+} \leftarrow \tilde{X}^{1} \Sigma^{+}$, but differs in the spin state. The HOMO-LUMO excitation $\left(\pi-\pi^{*}\right)$ provides three excited states, $\Sigma^{+}, \Sigma^{-}$, and $\Delta[12,35]$. Among the three, the upper state for the fully allowed transition, ${ }^{1} \Sigma^{+}$, locates at the highest energy and the other two, ${ }^{1} \Sigma^{-}$and ${ }^{1} \Delta$, come to the lowest excited energy within the singlet manifold. For the symmetry forbidden transition to the ${ }^{1} \Delta$ state from the $\tilde{X}^{1} \Sigma^{+}$ground state, Herzberg-Teller coupling with a vibrational $\pi$-mode excitation is crucial. The lowest energy triplet state for the phosphorescence, $\tilde{a}^{3} \Sigma^{+}$, locates slightly lower than the lowest energy singlets, thus the phosphorescence excitation measurement for the ${ }^{1} \Delta$ state is possible [12]. The vibronic structure in the phosphorescence excitation spectrum for the ${ }^{1} \Delta \leftarrow \tilde{X}^{1} \Sigma^{+}$transition is much simpler than the structure for the fully allowed ${ }^{1} \Sigma^{+} \leftarrow \tilde{X}^{1} \Sigma^{+}$transition.

\section{Figure 11.}

Since the upper state of the former is remote in energy from the upper state of the fully allowed transition, the transition occurs simply within a single pair of the upper and lower electronic states, even though the Herzberg-Teller coupling is required [12,32-34]. In contrast, the fully allowed transition of the latter occurs with numerous vibronic 
transitions to a number of nearby electronic states to which the intensity borrowing from the fully allowed transition is heavily promoted, i.e., the oscillator strength of the fully allowed transition is distributed to the other electronic transitions having overlapping vibronic levels in the vicinity. In this type of strongly coupled plasmonic transitions, the vibronic intensity is not simply governed by usual Franck-Condon factors between the two electronic states and even the origin band is not easily located [37].

\section{Summary}

Two cyanopolyyne molecules, $\mathrm{HC}_{9} \mathrm{~N}$ and $\mathrm{HC}_{11} \mathrm{~N}$, were isolated from solutions of laser-ablated graphite particles in acetonitrile and UV absorption spectra were recorded in acetonitrile and $n$-hexane. IR absorption spectra were measured in $\mathrm{CCl}_{4}$, while UV resonance Raman spectra were observed in $n$-hexane. Appearing both in the IR and Raman spectra in a range of $2030-2240 \mathrm{~cm}^{-1}$, five modes in $\mathrm{HC}_{9} \mathrm{~N}, \sigma_{2}-\sigma_{6}$, and five modes in $\mathrm{HC}_{11} \mathrm{~N}, \sigma_{2}-\sigma_{7}$ except for $\sigma_{4}$, were identified as stretching vibrational modes of the sp-hybridized $-(\mathrm{C} \equiv \mathrm{C}-)_{n} \mathrm{C} \equiv \mathrm{N}$ chain. Phosphorescence signals appearing at 582.3 $\mathrm{nm}(0-0), 665.4 \mathrm{~nm}(0-1)$, and $775.6 \mathrm{~nm}(0-2)$, and $927.8 \mathrm{~nm}(0-3)$ in the solid acetonitrile matrix host constitute vibrational progression of the stretching $\sigma_{4}$ mode in the electronic ground state of $\mathrm{HC}_{9} \mathrm{~N}$, fairly consistent with the vibrational frequency of $2141 \mathrm{~cm}^{-1}$ by the IR and Raman measurement. For $\mathrm{HC}_{11} \mathrm{~N}$, phosphorescence signals were peaking at $643.7 \mathrm{~nm}(0-0), 744.8 \mathrm{~nm}(0-1)$, and $881.7 \mathrm{~nm}(0-2)$, consistent with the $\sigma_{6}$ fundamental of $2105 \mathrm{~cm}^{-1}$ in the IR and Raman spectra. Phosphorescence excitation mapping showed UV absorption dynamics in the cryogenic solids of organic molecules explainable by the presence of multiple trapping sites as well as crystalline sites with sharp emission/absorption features by Shpolsky effects, i.e., the three distinct trapping sites for $\mathrm{HC}_{9} \mathrm{~N}$ in solid acetonitrile in particular. Vibronic patterns of the UV excitation were found irregular which highlight the multiple electronic excited states involved. Phosphorescence lifetimes were longer for $\mathrm{HC}_{9} \mathrm{~N}$ than that for $\mathrm{HC}_{11} \mathrm{~N}$.

\section{Acknowledgments}


The MEXT-Supported Program for the Strategic Research Foundation at Private Universities for Establishing a Best-Energy-Mix Research Center to Promote the Use of Solar Energy subsidized from the Ministry of Education, Culture, Sports, Science and Technology of Japan (MEXT) and Kindai University is greatly acknowledged. US is supported by the Mobility Contest Internship Program, Institute of Physical Chemistry, Polish Academy of Sciences for collaboration performed at Kindai University.

\section{Supplementary data}

Calculated IR and Raman data are provided as supplementary Table S1 with their plots in spectra in Figs. S1 (IR) and S2 (Raman) for $\mathrm{HC}_{9} \mathrm{~N}$ and $\mathrm{HC}_{11} \mathrm{~N}$. Molecular orbital (MO) calculations were performed at the B3LYP level of density functional theory (DFT) with various basis sets using M. J. Frisch et al., Gaussian 16, Revision A.03, Gaussian Inc. Wallingford, CT, 2016. The calculated harmonic frequencies are scaled by 0.967 for anharmonicity corrections.

\section{References}

[1] N.W. Broten, T. Oka, L.W. Avery, J.M. MacLeod, H.W. Kroto, The detection of $\mathrm{HC}_{9} \mathrm{~N}$ in interstellar space, Astrophys. J. 223 (1978) L105.

[2] R.L. Snell, F.P. Schloerb, J.S. Young, A. Hjalmarson, P. Friberg, Observations of $\mathrm{HC}_{3} \mathrm{~N}, \mathrm{HC}_{5} \mathrm{~N}$, and $\mathrm{HC}_{7} \mathrm{~N}$ in molecular clouds, Astrophys. J. 244 (1981) 45-53.

[3] M.B. Bell, L.W. Avery, J.M. MacLeod, H.E. Matthews, The excitation temperature of $\mathrm{HC}_{9} \mathrm{~N}$ in the circumstellar envelope of IRC +10216 , Astrophys. J. 400 (1992) 551-555.

[4] Truong-Bach, D. Graham, Nguyen-Q-Rieu, $\mathrm{HC}_{9} \mathrm{~N}$ from the Envelopes of IRC+10216 and CRL:2688, Astron. Astrophys. 277 (1993) 133.

[5] R.A. Loomis, C.N. Shingledecker, G. Langston, B.A. McGuire, N.M. Dollhopf, A.M. Burkhardt, J. Corby, S.T. Booth, P.B. Carroll, B. Turner, A.J. Remijan, 
Non-detection of $\mathrm{HC}_{11} \mathrm{~N}$ towards TMC-1: Constraining the chemistry of large carbon-chain molecules, Mon. Not. R. Astron. Soc. 463 (2016) 4175-4183.

[6] K.M.T. Yamada, M. Bester, M. Tanimoto, G. Winnewisser, Pure rotational spectrum of cyanopropyne in the $\mathrm{v}_{12}=1$ vibrational state, J. Mol. Spectrosc. 126 (1987) 118-128.

[7] A. Coupeaud, R. Kołos, I. Couturier-Tamburelli, J.P. Aycard, N. Piétri, Photochemical Synthesis of the Cyanodiacetylene $\mathrm{HC}_{5} \mathrm{~N}$ : A Cryogenic Matrix Experiment, J. Phys. Chem. A 110 (2006) 2371-2377.

[8] C. Crépin, M. Turowski, J. Ceponkus, S. Douin, S. Boyé-Péronne, M. Gronowski, R. Kołos, UV-Induced growth of cyanopolyyne chains in cryogenic solids, Phys. Chem. Chem. Phys. 13 (2011) 16780-16785.

[9] I. Couturier-Tamburelli, N. Piétri, C. Crépin, M. Turowski, J.-C. Guillemin, R. Kołos, Synthesis and spectroscopy of cyano-triacetylene $\left(\mathrm{HC}_{7} \mathrm{~N}\right)$ in solid argon, $\mathrm{J}$. Chem. Phys. 140 (2014) 044329.

[10]M. Turowski, C. Crépin, M. Gronowski, J.-C. Guillemin, A. Coupeaud, I. Couturier-Tamburelli, N. Piétri, R. Kołos, Electronic absorption and phosphorescence of cyanodiacetylene, J. Chem. Phys. 133 (2010) 074310.

[11]M. Turowski, C. Crépin, S. Douin, M. Gronowski, I. Couturier-Tamburelli, N. Piétri, A. Wasiak, R. Kołos, Low temperature Raman spectra of cyanobutadiyne $\left(\mathrm{HC}_{5} \mathrm{~N}\right)$, Vib. Spectrosc. 62 (2012) 268-272.

[12]U. Szczepaniak, C. Crépin, M. Gronowski, M. Chevalier, J.-C. Guillemin, M. Turowski, T. Custer, R. Kołos, Cryogenic photochemical synthesis and electronic spectroscopy of cyanotetraacetylene. J. Phys. Chem. A 121 (2017) 7374-7384.

[13]U. Szczepaniak, M. Turowski, T. Custer, M. Gronowski, N. Kerisit, Y. Trolez, R. Kołos, Infrared and Raman spectroscopy of methylcyanodiacetylene $\left(\mathrm{CH}_{3} \mathrm{C}_{5} \mathrm{~N}\right)$, ChemPhysChem 17 (2016) 3047-3054.

[14]U. Szczepaniak, R. Kołos, M. Gronowski, M. J.-C. Guillemin, C. Crépin, Low temperature synthesis and phosphorescence of methyltriacetylene, J. Phys. Chem. A 122 (2018) 89-99.

[15]M. Turowski, U. Szczepaniak, T. Custer, M. Gronowski, R. Kołos, Electronic spectroscopy of methylcyanodiacetylene $\left(\mathrm{CH}_{3} \mathrm{C}_{5} \mathrm{~N}\right)$, ChemPhysChem 17 (2016) 4068-4078. 
[16]H. Tabata, M. Fujii, M. Hayashi, T. Doi, T. Wakabayashi, Raman and surface-enhanced Raman scattering of a series of polyynes, Carbon 44 (2006) 3168-3176.

[17]T. Wakabayashi, M. Saikawa, Y. Wada, T. Minematsu, Isotope scrambling in the formation of cyanopolyynes by laser ablation of carbon particles in liquid acetonitrile, Carbon 50 (2012) 47-56.

[18]T. Wakabayashi, H. Tabata, T. Doi, H. Nagayama, K. Okuda, R. Umeda, I. Hisaki, M. Sonoda, Y. Tobe, T. Minematsu, K. Hashimoto, S. Hayashi, Resonance Raman spectra of polyyne molecules $\mathrm{C}_{10} \mathrm{H}_{2}$ and $\mathrm{C}_{12} \mathrm{H}_{2}$ in solution, Chem. Phys. Lett. 433 (2007) 296-300.

[19]T. Wakabayashi, H. Nagayama, K. Daigoku, Y. Kiyooka, K. Hashimoto, Laser induced emission spectra of polyyne molecules $\mathrm{C}_{2 n} \mathrm{H}_{2}(n=5-8)$, Chem. Phys. Lett. 446 (2007) 65-70.

[20] Y. Wada, Y. Morisawa, T. Wakabayashi, Spectroscopic characterization of a series of polyyne-iodine molecular complexes $\mathrm{H}(\mathrm{C} \equiv \mathrm{C})_{n} \mathrm{H}\left(\mathrm{I}_{6}\right)$ of $n=5-9$, Chem. Phys. Lett. 541 (2012) 54-59.

[21]D. Nishide, H. Dohi, T. Wakabayashi, E. Nishibori, S. Aoyagi, M. Ishida, S. Kikuchi, R. Kitaura, T. Sugai, H. Shinohara, Single-wall carbon nanotubes encaging linear chain $\mathrm{C}_{10} \mathrm{H}_{2}$ polyyne molecules inside, Chem. Phys. Lett. 428 (2006) 356-360.

[22]D. Nishide, T. Wakabayashi, H. Kataura, Y. Achiba, H. Shinohara, Raman spectroscopy of size-selected linear polyyne molecules $\mathrm{C}_{2 n} \mathrm{H}_{2} \quad(n=4-6)$ encapsulated in single-wall carbon nanotubes, J. Phys. Chem. C 111 (2007) 5178-5183.

[23]T. Wakabayashi, T. Murakami, H. Nagayama, D. Nishide, H. Kataura, Y. Achiba, H. Tabata, S. Hayashi, H. Shinohara, Raman spectral features of longer polyynes $\mathrm{HC}_{2 n} \mathrm{H}(n=4-8)$ in SWNTs, Eur. Phys. J. D 52 (2009) 79-82.

[24]R. Sata, H. Suzuki, N. Ueno, Y. Morisawa, M. Hatanaka, T. Wakabayashi, UV-polarizing linear polyyne molecules aligned in PVA, Chin. J. Chem. Phys. 32 (2019) 175-181.

[25]Z. Mielke, A. Olbert-Majkut, K. G. Tokhadze, Photolysis of the OC $\cdots$ HONO complex in low temperature matrices: Infrared detection and ab initio calculations of nitrosoformic acid, HOC(O)NO, J. Chem. Phys. 118 (2003) 1364-1377. 
[26]Z. Mielke, S. Coussan, K. Mierzwicki, P. Roubin, M. Sałdyka, The complex between $\mathrm{CH} 3 \mathrm{OH}$ and $\mathrm{CF} 4$. Infrared matrix isolation and theoretical studies, J. Phys. Chem. A 110 (2006) 4712-4718.

[27]M. Sałdyka and Z. Mielke, The interaction of formohydroxamic acid with nitrogen: FTIR matrix isolation and ab initio studies, J. Mol. Struct. 708 (2004) 183-188.

[28]R. Eastmond, T. R. Johnson, D. R. M. Walton, Silylation as a protective method for terminal alkynes in oxidative couplings - A general synthesis of the parent polyynes $\mathrm{H}(\mathrm{C} \equiv \mathrm{C}){ }_{n} \mathrm{H}(n=4-10,12)$, Tetrahedron 28 (1972) 4601-4616.

[29]T. Wakabayashi, T. Momose, M. E. Fajardo, Matrix isolation spectroscopy and spectral simulations of isotopically substituted $\mathrm{C}_{60}$ molecules, J. Chem. Phys. 151 (2019) 234301.

[30]H. Hoshina, T. Wakabayashi, T. Momose, T. Shida, Infrared spectroscopic study of rovibrational states of perdeuterated methane $\left(\mathrm{CD}_{4}\right)$ trapped in parahydrogen crystal, J. Chem. Phys. 110 (1999) 5728-5733.

[31]T. Momose, H. Hoshina, N. Sogoshi, H. Katsuki, T. Wakabayashi, T. Shida, Tunneling chemical reactions in solid parahydrogen: A case of $\mathrm{CD}_{3}+\mathrm{H}_{2} \rightarrow \mathrm{CD}_{3} \mathrm{H}$ + H at 5 K, J. Chem. Phys. 108 (1998) 7334-7338.

[32]G. Orlandi and W. Siebrand, Theory of vibronic intensity borrowing. Comparison of Herzberg-Teller and Born-Oppenheimer coupling, J. Chem. Phys. 58 (1973) 4513-4523.

[33] S. H. Lin and H. Eyring, Study of vibronic and Born-Oppenheimer couplings, Proc. Nat. Acad. Sci. USA 71 (1974) 3415-3417.

[34]S. H. Lin and H. Eyring, Study of the Franck-Condon and Herzberg-Teller Approximations, Proc. Nat. Acad. Sci. USA 71 (1974) 3802-3804.

[35]T. Wakabayashi, Y. Wada, N. Iwahara, T. Sato, Vibronic bands in the HOMO-LUMO excitation of linear polyyne molecules, J. Phys: Conf. Ser. 428 (2013) 012004.

[36]A. V. Naumov, Low-temperature spectroscopy of organic molecules in solid matrices: from the Shpol'skii effect to laser luminescent spectroscopy for all effectively emitting single molecules, Phys. Usp. 56 (2013) 605-622 [Usp. Fiz. Nauk. 183 (2013) 633-652].

[37]G. Monninger, M. Förderer, P. Gürtler, S. Kalhofer, S. Petersen, L. Nemes, P. G. Szalay, W. Krätschmer, Vacuum ultraviolet spectroscopy of the carbon molecule 
$\mathrm{C}_{3}$ in matrix isolated state: Experiment and theory, J. Phys. Chem. 106 (2002) 5779-5788. 
Table 1. UV absorption and phosphorescence excitation features of $\mathrm{HC}_{9} \mathrm{~N}$ and $\mathrm{HC}_{11} \mathrm{~N}$.

\begin{tabular}{|c|c|c|c|c|c|}
\hline \multirow{2}{*}{ Species } & \multicolumn{2}{|c|}{ Absorption / nm } & \multicolumn{3}{|c|}{ Phosphorescence Excitation / nm } \\
\hline & Acetonitrile $^{a)}$ & $n$-Hexane ${ }^{\text {a) }}$ & Acetonitrile $^{\mathrm{b})}$ & $n$-Hexane $\mathrm{e}^{\mathrm{b})}$ & $\mathrm{Kr}^{\mathrm{c})}$ \\
\hline \multirow[t]{11}{*}{$\mathrm{HC}_{9} \mathrm{~N}$} & 201.3 & & & & \\
\hline & 211.8 & 212.0 & & & $\begin{array}{l}210.4[47,534] \\
211.9[47,201]\end{array}$ \\
\hline & 221.6 & 221.0 & $223(224)$ & 222 & $221.0[45,247]$ \\
\hline & 230.9 & 231.0 & $232(235)$ & 231 & $231.5[43,189]$ \\
\hline & 238.8 & 238.8 & 240.7 & 241 & $238.2[41,973]$ \\
\hline & 244.4 & 245.6 & $(244.9)$ & & $244.0[40,986]$ \\
\hline & 248.9 & 248.6 & 249.6 & 250 & $248.4[40,252]$ \\
\hline & & 255.0 & $(255)$ & 255 & $255.9[39,085]$ \\
\hline & 260.3 & 261.0 & $263(267)$ & 262 & $260.7[38,358]$ \\
\hline & 273.7 & 272.0 & $275(278)$ & 274 & $\begin{array}{l}272.9[36,647] \\
273.6[36,554]\end{array}$ \\
\hline & 288.3 & & & & $288.1[34,706]$ \\
\hline \multirow{12}{*}{$\mathrm{HC}_{11} \mathrm{~N}$} & 2128 & 12 & & & \\
\hline & 2222 & 2222 & 224.25 & & \\
\hline & 232.1 & 232.0 & 233.75 & & \\
\hline & 242.0 & 242.2 & 244.50 & & \\
\hline & 253.3 & 253.3 & 255.25 & & \\
\hline & & & 263.25 & & \\
\hline & 204.5 & 204.4 & 267.00 & & \\
\hline & 274.5 & & 275.75 & & \\
\hline & & 284.0 & & & \\
\hline & 289.2 & 287.0 & 289.75 & & \\
\hline & 304.9 & 303.8 & & & \\
\hline & 321.8 & & & & \\
\hline
\end{tabular}

a) Solutions at ambient temperature [17].

b) Solid matrices at $20 \mathrm{~K}$ : Wavelengths in parenthesis are for Site B in solid acetonitrile (this work).

c) Solid Kr matrices: Wavenumbers in $\mathrm{cm}^{-1}$ in braces are reported in the literature [12]. 
Table 2. Observed IR and Raman peaks of $\mathrm{HC}_{9} \mathrm{~N}$ and $\mathrm{HC}_{11} \mathrm{~N}$ in solutions.

\begin{tabular}{|c|c|c|c|c|c|}
\hline \multirow{2}{*}{ Species } & \multirow{2}{*}{$\begin{array}{l}\text { Frequency }\left(\mathrm{cm}^{-1}\right) \\
\qquad \mathrm{IR}^{\mathrm{b})}\end{array}$} & \multicolumn{2}{|c|}{ Intensity $^{a}$} & \multirow{2}{*}{ Mode } & \multirow{2}{*}{ Assignment $^{\mathrm{g})}$} \\
\hline & & $\mathrm{IR}^{\mathrm{b})}$ & $\operatorname{Raman}^{\mathrm{c})}$ & & \\
\hline \multirow[t]{9}{*}{$\mathrm{HC}_{9} \mathrm{~N}$} & 3302 & s. & & $\sigma_{1}$ & CH str. \\
\hline & 2239 & $\mathrm{~m}$. & w. & $\sigma_{2}$ & CN str. ${ }^{\text {h) }}$ \\
\hline & 2190 & vw. & vw. & $\sigma_{3}$ & \\
\hline & 2174 & w. & w. & $2 \sigma_{8}$ & \\
\hline & 2141 & s. & s. & $\sigma_{4}$ & - CC str. ${ }^{\mathrm{i})}$ \\
\hline & 2115 & w. & w. & $\sigma_{5}$ & \\
\hline & 2030 & - & w. ${ }^{\text {d) }}$ & $\sigma_{6}$ & \\
\hline & 632 & sh. & & $\pi$ & $\mathrm{CH}$ hend \\
\hline & 626 & s. & & & - \\
\hline \multirow[t]{9}{*}{$\mathrm{HC}_{11} \mathrm{~N}$} & 3303 & s. & & $\sigma_{1}$ & $\mathrm{CH}$ str. \\
\hline & 2235 & $\mathrm{~m}$. & w. & $\sigma_{2}$ & CN str. \\
\hline & 2184 & vw. & - & $\sigma_{3}$ & \\
\hline & 2139 & W. & - & $\sigma_{5}^{\mathrm{e})}$ & \\
\hline & 2105 & s. & S. & $\int \sigma_{6}{ }^{\mathrm{e})}$ & - CC str. \\
\hline & $2100]$ & & & {$\left[\sigma_{7}+2 \pi_{11}\right.$} & \\
\hline & 2085 & w. & sh. & combination $^{\text {f) }}$ & \\
\hline & 2028 & - & w. & $\sigma_{7}$ & \\
\hline & 632 & $\mathrm{~m}$. & & $\pi_{1}$ & $\mathrm{CH}$ bend. \\
\hline
\end{tabular}

a) Abbreviations: s. (strong), m. (medium), w. (weak), vw. (very weak), sh. (shoulder).

b) Solvent: $\mathrm{CCl}_{4}$.

c) Solvent: $n$-hexane.

d) Overlapping with an impurity peak.

e) Strongly perturbed by overtones and combinations.

f) Candidates: $\sigma_{9}+4 \pi_{8}, 2 \sigma_{11}+2 \pi_{6}$, or $\sigma_{9}+\sigma_{10}$.

g) Abbreviations: str. (stretching), bend. (bending).

h) A collective mode with CC stretching motions.

i) Collective modes with the $\mathrm{CN}$ stretching motion. 
Table 3. Major vibrational progression observed in the dispersed phosphorescence spectra of $\mathrm{HC}_{9} \mathrm{~N}$ and $\mathrm{HC}_{11} \mathrm{~N}$ in cryogenic matrix hosts of solid organic molecules.

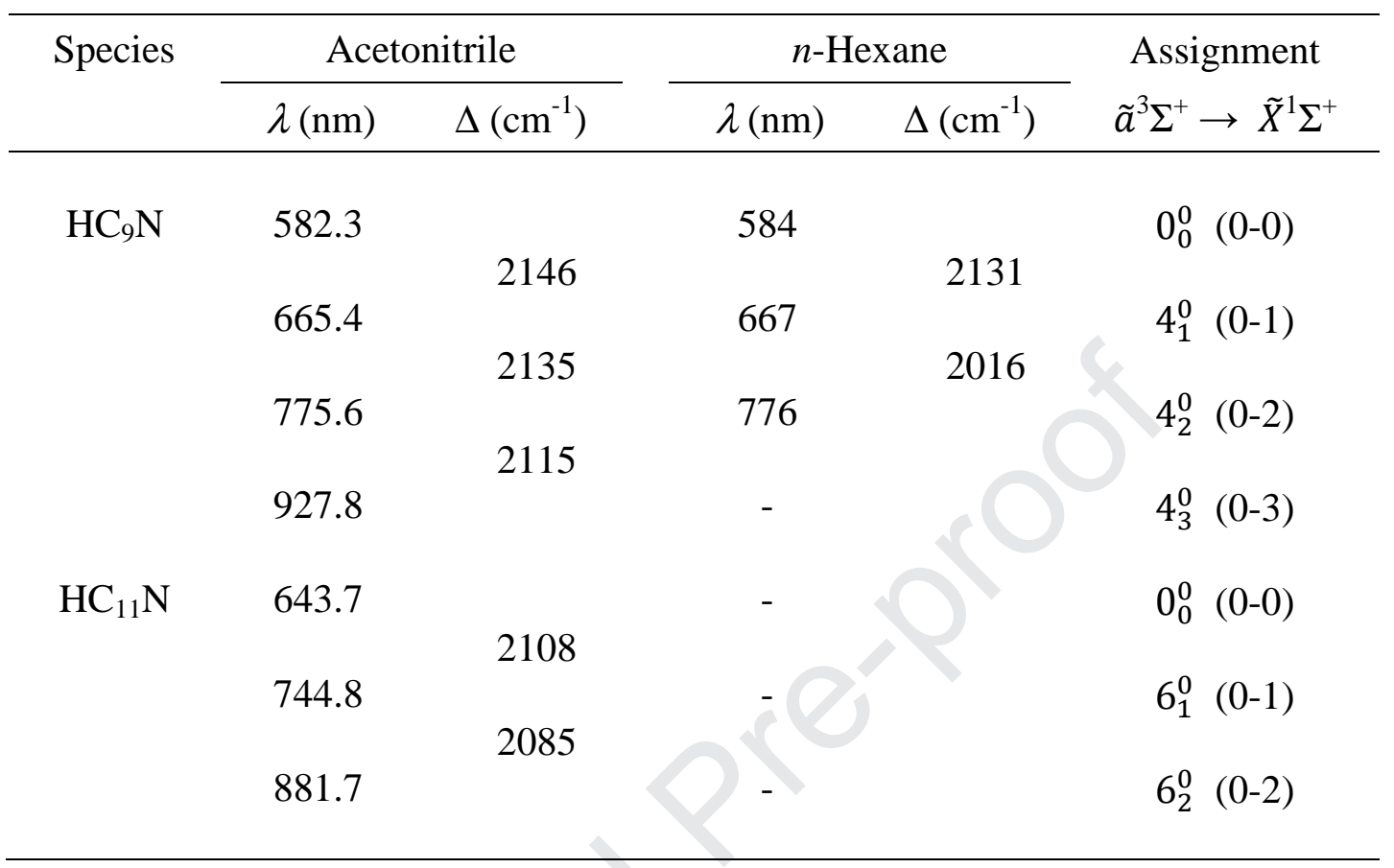


Table 4. Site peaks and satellite peaks in the phosphorescence spectra of $\mathrm{HC}_{9} \mathrm{~N}$ in solid acetonitrile at $20 \mathrm{~K}$. Three distinct matrix sites are denoted by A, B, and C. Difference frequencies in $\mathrm{cm}^{-1}$ are relative to the main peak within the same group of $0-\mathrm{v}$ bands $(\mathrm{v}$ $=0,1$, and 2) in the common site.

\begin{tabular}{|c|c|c|c|c|c|c|c|}
\hline \multirow{3}{*}{$\begin{array}{c}\mathrm{HC}_{9} \mathrm{~N} \\
\text { Progression } \\
\sigma_{4}\end{array}$} & \multicolumn{4}{|c|}{ Observed Peaks } & \multirow{2}{*}{\multicolumn{3}{|c|}{$\frac{\text { Difference Frequencies }}{\text { Wavenumber } / \mathrm{cm}^{-1}}$}} \\
\hline & \multirow{2}{*}{ Appearance } & \multicolumn{3}{|c|}{ Wavelength / nm } & & & \\
\hline & & $\mathrm{A}$ & $\mathrm{B}$ & $\mathrm{C}$ & $\mathrm{A}$ & $\mathrm{B}$ & $\mathrm{C}$ \\
\hline \multirow{6}{*}{$0-0$} & satellite & 579.6 & - & - & -78 & - & - \\
\hline & shoulder & 582.1 & - & - & -4 & - & - \\
\hline & main peak & 582.3 & 593.9 & 598.7 & 0 & 0 & 0 \\
\hline & satellite $^{\text {a) }}$ & 583.4 & - & - & 32 & - & - \\
\hline & shoulder & 584.7 & - & 600.2 & 72 & - & 42 \\
\hline & weak band ${ }^{\text {b) }}$ & - & 611.4 & 617.6 & - & 482 & 510 \\
\hline \multirow{7}{*}{$0-1$} & shoulder & 660.6 & 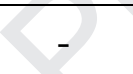 & - & -109 & - & - \\
\hline & satellite & 661.8 & - & - & -83 & - & - \\
\hline & shoulder & 664.3 & - & - & -24 & - & - \\
\hline & main peak & 665.4 & 681.1 & 687.4 & $2146^{\mathrm{c})}$ & $2156^{\mathrm{c})}$ & $2155^{\mathrm{c})}$ \\
\hline & satellite $^{a)}$ & 666.9 & - & - & 33 & - & - \\
\hline & shoulder & 668.5 & 682.4 & 689.7 & 69 & 28 & 48 \\
\hline & weak band ${ }^{\text {b) }}$ & - & 703.8 & 710.9 & - & 473 & 480 \\
\hline \multirow{5}{*}{$0-2$} & satellite & 769.8 & - & - & -97 & - & - \\
\hline & shoulder & 774.4 & - & - & -20 & - & - \\
\hline & main peak & 775.6 & 797.6 & 805.5 & $2135^{\mathrm{d})}$ & $2144^{\mathrm{d})}$ & $2132^{\mathrm{d})}$ \\
\hline & satellite $^{\text {a) }}$ & 777.5 & - & - & 30 & - & - \\
\hline & shoulder & 779.8 & - & - & 69 & - & - \\
\hline $0-3$ & main peak & 927.8 & 960.8 & - & $2115^{\mathrm{e})}$ & $2130^{\mathrm{e})}$ & - \\
\hline
\end{tabular}

a) Split side-peaks in Fig. 3 and a down-shifted peak "a" in Fig. 4.

b) Assigned to $v \sigma_{4}+\pi_{4}(\mathrm{v}=0,1$, and 2) as in Ref. 12 .

c) Difference from the $0-0$ main peak.

d) Difference from the 0-1 main peak.

e) Difference from the 0-2 main peak. 


\section{Figure Captions}

Figure 1. UV absorption spectra of $\mathrm{HC}_{9} \mathrm{~N}$ and $\mathrm{HC}_{11} \mathrm{~N}$ in solutions of $n$-hexane.

Figure 2. IR absorption spectra in $\mathrm{CCl}_{4}$ (upper traces) and UV resonance Raman spectra in $n$-hexane (lower traces) of $\mathrm{HC}_{9} \mathrm{~N}$ and $\mathrm{HC}_{11} \mathrm{~N}$ in solutions at ambient temperature. A peak with an asterisk is a Raman signal of impurity molecules.

Figure 3. Dispersed phosphorescence spectra of $\mathrm{HC}_{9} \mathrm{~N}$ in solid acetonitrile $\left(\mathrm{CH}_{3} \mathrm{CN}\right)$ at $20 \mathrm{~K}$. a) Spectra in $\mathrm{nm}$ at different excitation wavelengths of 250.0, 255.0, and $271.0 \mathrm{~nm}$ and $\mathrm{b}$ ) the spectrum in $\mathrm{cm}^{-1}$ (background subtracted) at $250.0 \mathrm{~nm}$ excitation.

Figure 4. Phosphorescence mapping for $\mathrm{HC}_{9} \mathrm{~N}$ in solid acetonitrile at $20 \mathrm{~K}$. Dispersed phosphorescence spectra by excitations at 241, 245, $271 \mathrm{~nm}$ (top). Excitation spectra by phosphorescence intensities at 582.3, 593.9, and $598.7 \mathrm{~nm}$ (right).

Figure 5. Phosphorescence excitation spectra of $\mathrm{HC}_{9} \mathrm{~N}$ in solid acetonitrile at $20 \mathrm{~K}$ plotted for intensities at A 582.3, B 593.9, and C $598.7 \mathrm{~nm}$ (mode 3). Dotted line spectra are reproduced from Fig. 4 with vertical scaling (mode 2). Dashed line is the UV absorption spectrum in a solution of acetonitrile at ambient temperature, for which circle markers indicate absorption features at 238.8, 244.4, and $248.9 \mathrm{~nm}$. An asterisk indicates the artificial peak by SHG-crystal switching. 
Figure 6. a) Phosphorescence mapping for $\mathrm{HC}_{9} \mathrm{~N}$ in solid $n$-hexane at $20 \mathrm{~K}$. The trace in white is a dispersed phosphorescence spectrum by the excitation at $250 \mathrm{~nm} . \mathrm{b}$ ) Excitation spectra plotted by the phosphorescence intensity at indicated wavelengths. UV absorption spectra in $n$-hexane and acetonitrile at ambient temperature (dashed lines) and an excitation spectrum in acetonitrile in Fig. 4 (dotted line) are shown for comparison.

Figure 7. Phosphorescence spectrum of $\mathrm{HC}_{11} \mathrm{~N}$ in solid acetonitrile at $20 \mathrm{~K}$.

Figure 8. Phosphorescence excitation spectrum of $\mathrm{HC}_{11} \mathrm{~N}$ in solid acetonitrile at $20 \mathrm{~K}$ by monitoring the intensity at $644 \mathrm{~nm}$ during the continuous UV wavelength scan. Dotted line shows absorption spectrum in the solution at ambient temperature.

Figure 9. Phosphorescence spectra (left panel) and their excitation mapping (right panel) for the $0-0$ band of $\mathrm{HC}_{11} \mathrm{~N}$ in solid acetonitrile at $20 \mathrm{~K}$.

Figure 10. Phosphorescence excitation spectrum of $\mathrm{HC}_{11} \mathrm{~N}$ in solid acetonitrile at $20 \mathrm{~K}$ by plotting the peak intensity at $644 \mathrm{~nm}$ in Fig. 9 for the Shpolsky site.

Figure 11. Phosphorescence transition wavelengths of the 0-0 band for cyanopolyynes $\mathrm{HC}_{2 n+1} \mathrm{~N}(n=2-5)$ in solid $\mathrm{Ar}[9,10,12]$ and in solid acetonitrile (this work). UV absorption maxima in solutions are plotted for reference $[16,17,28]$. 

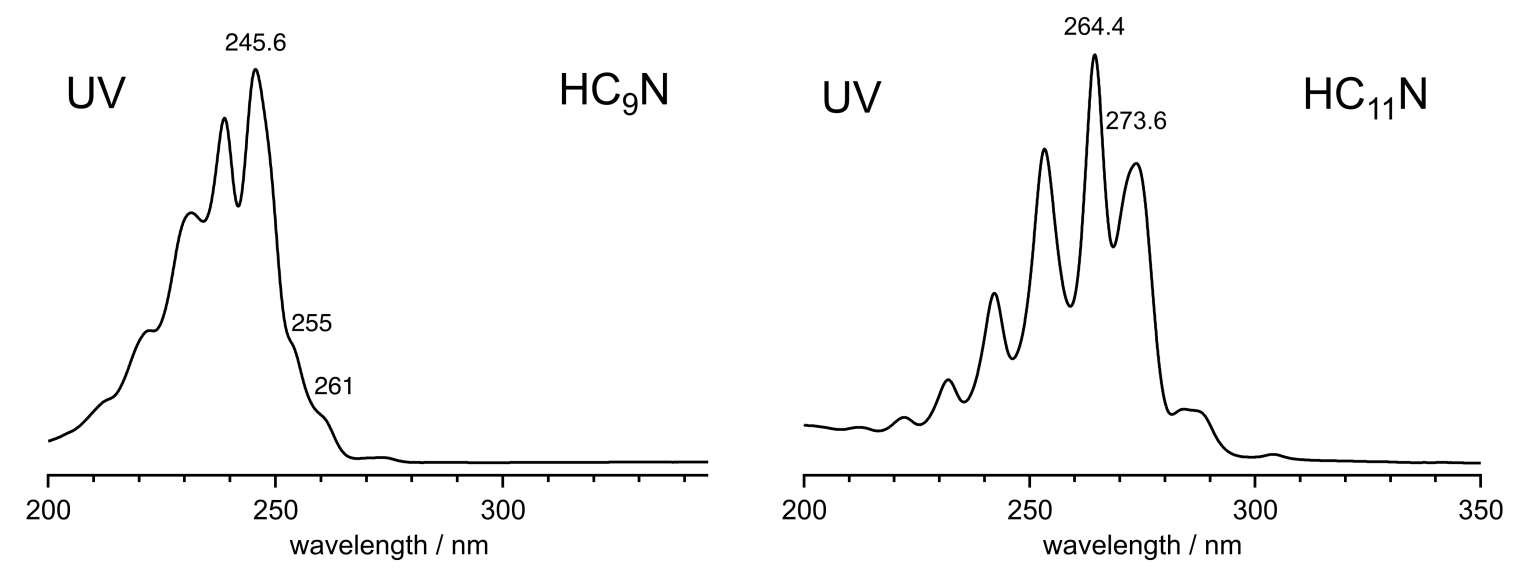


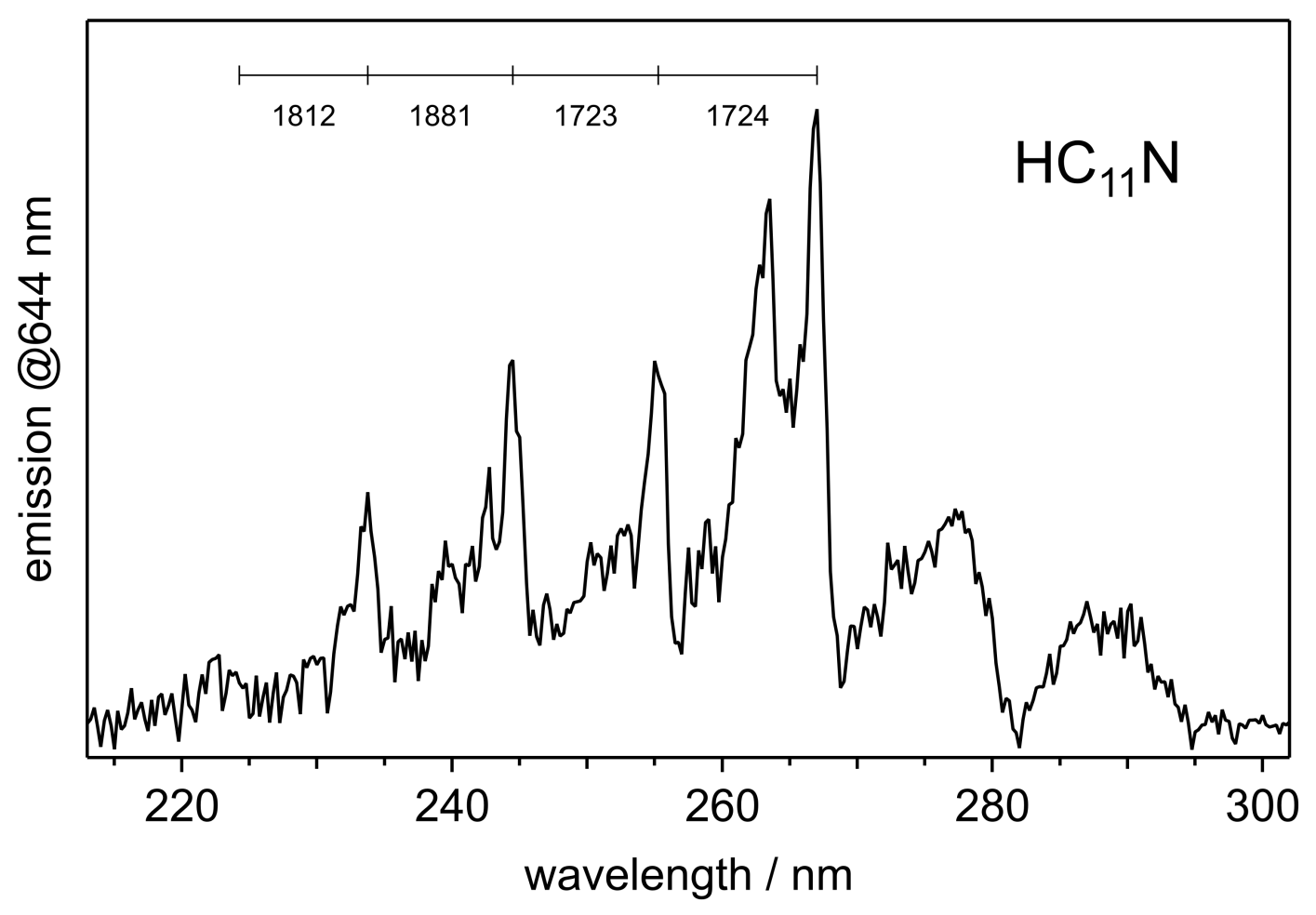




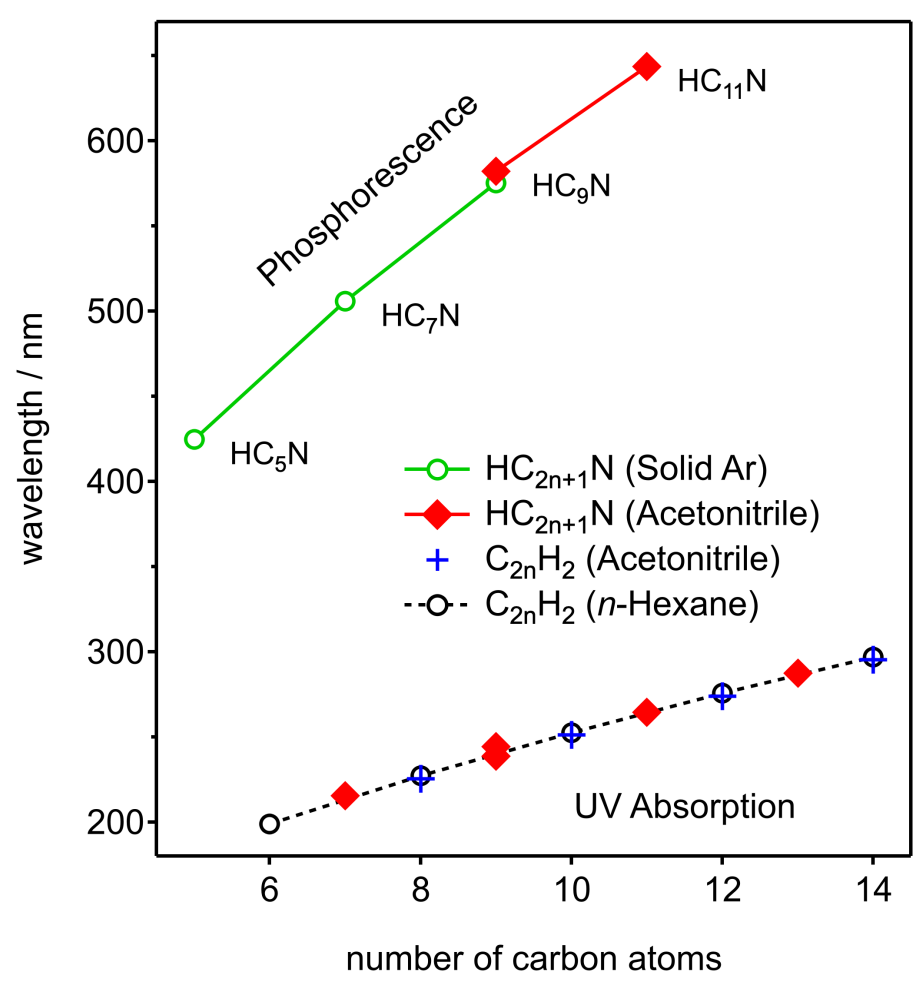



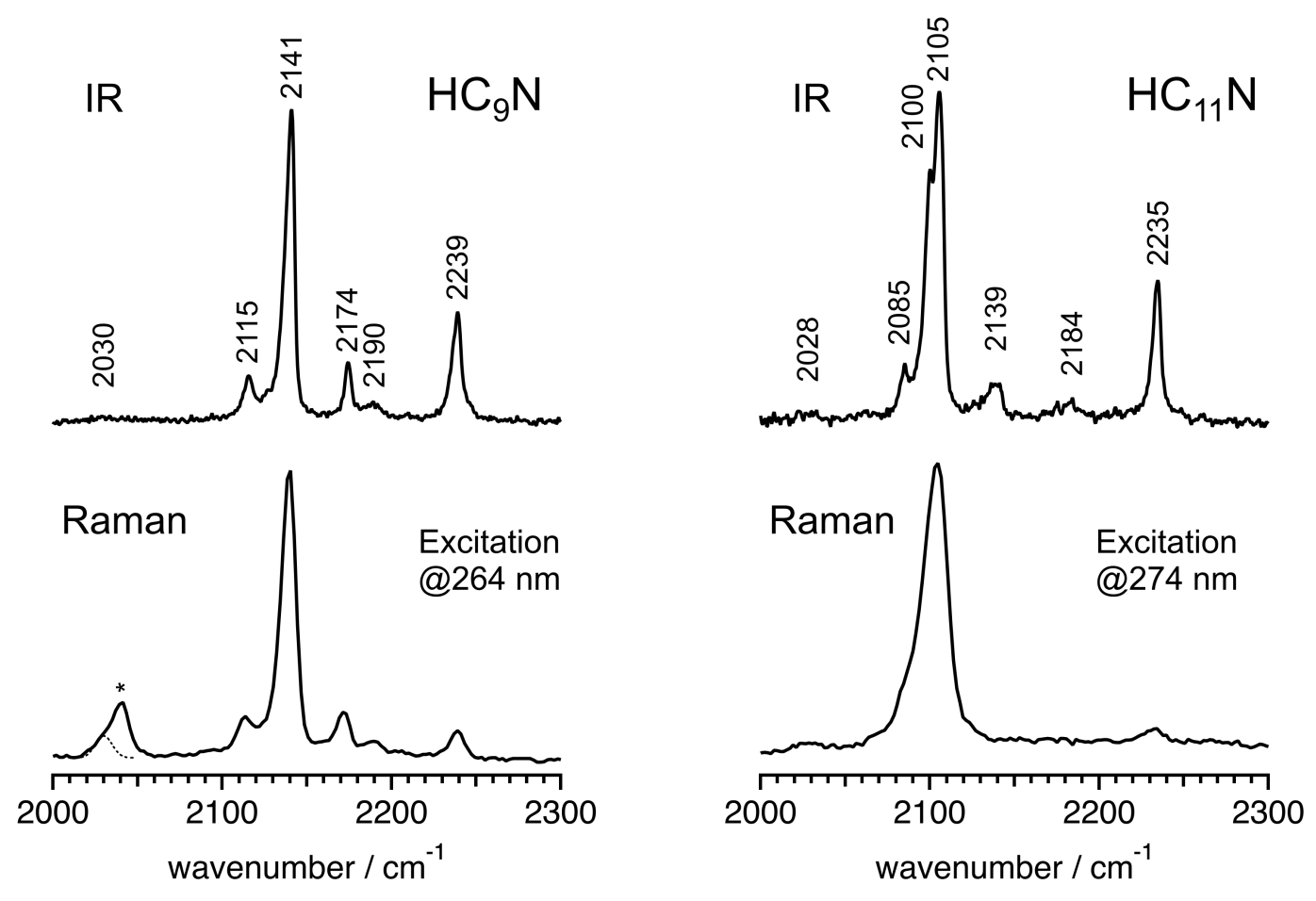
582.3

a)

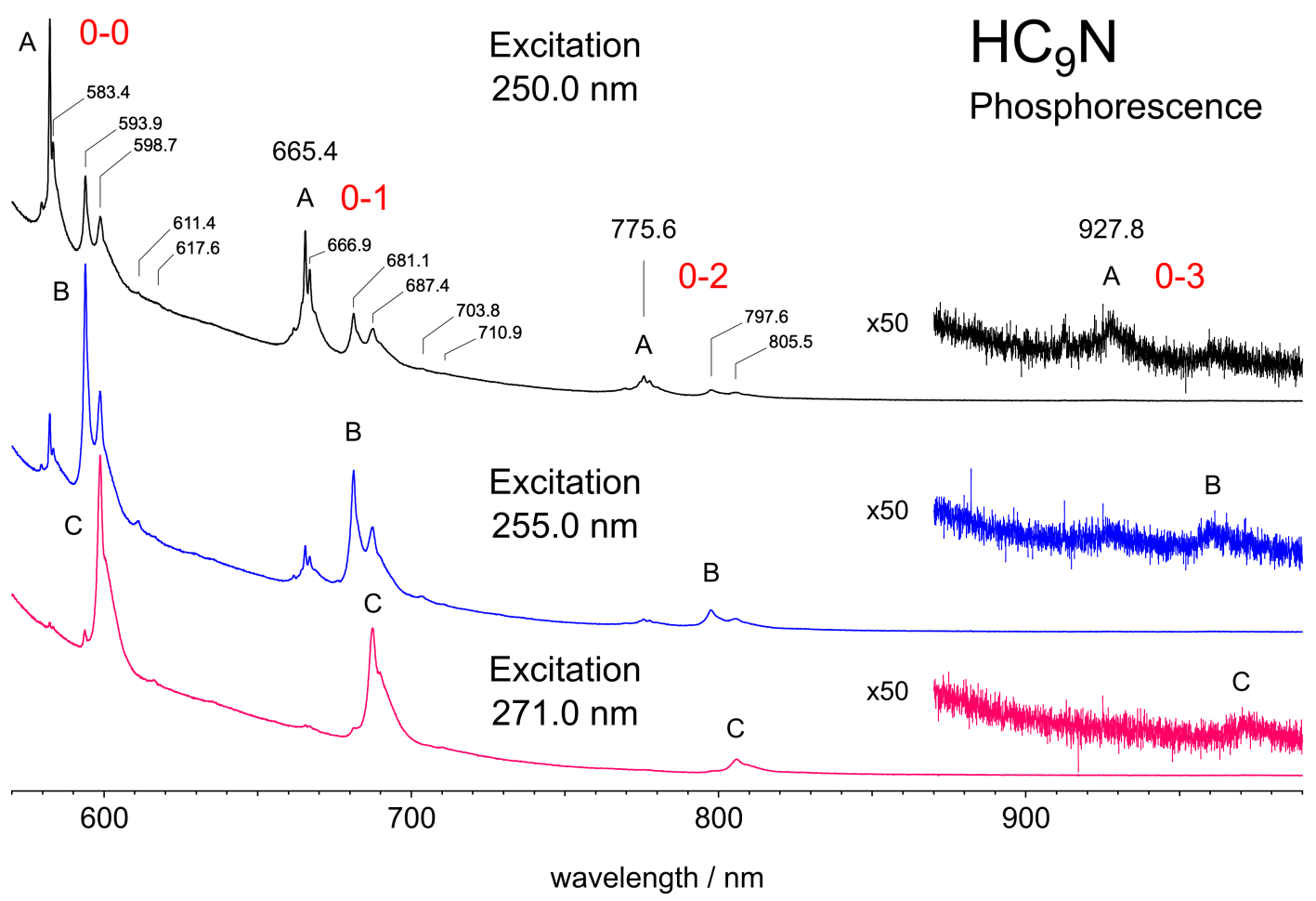

b)

$0^{0}{ }_{0}^{A}$

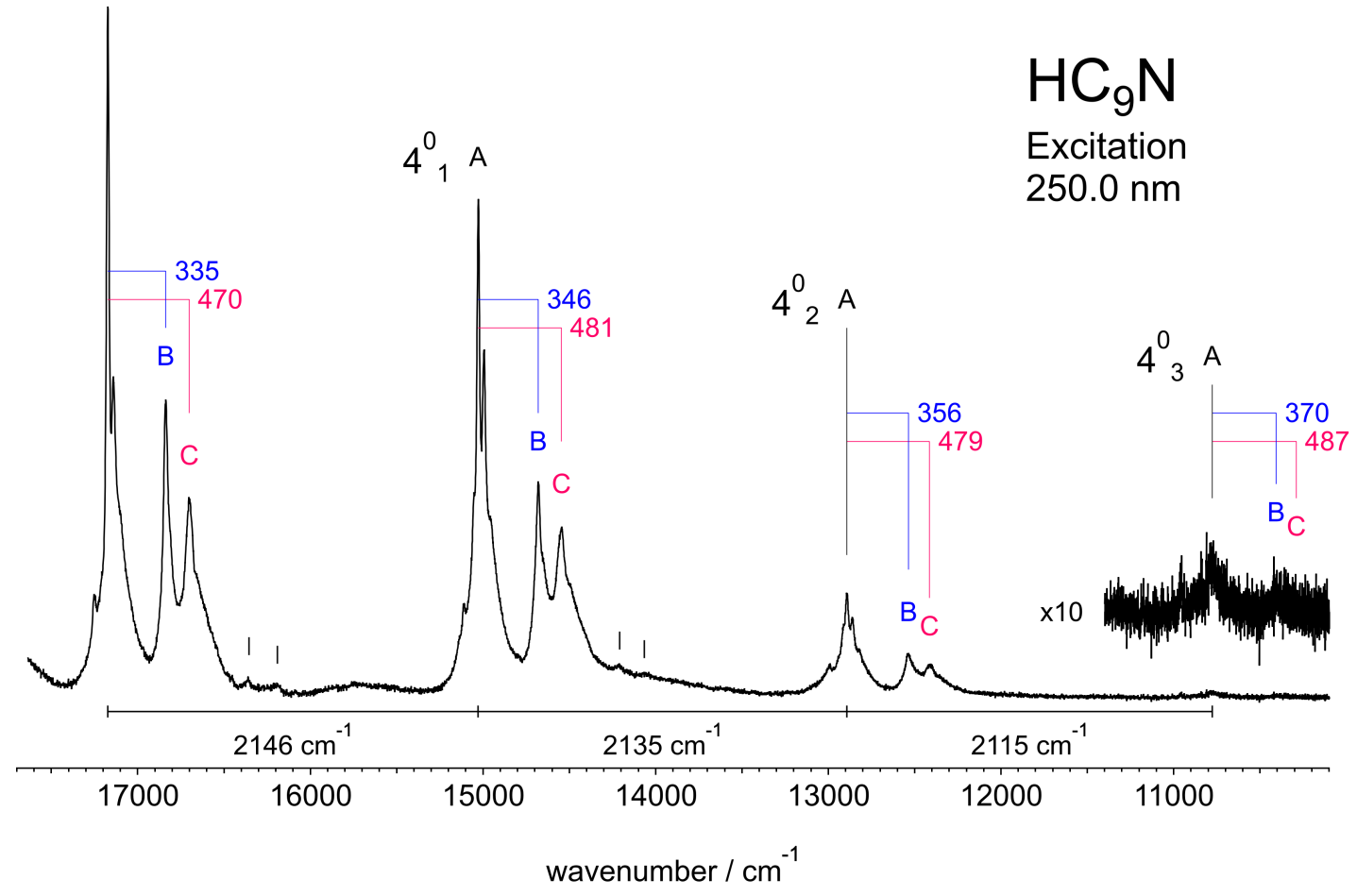




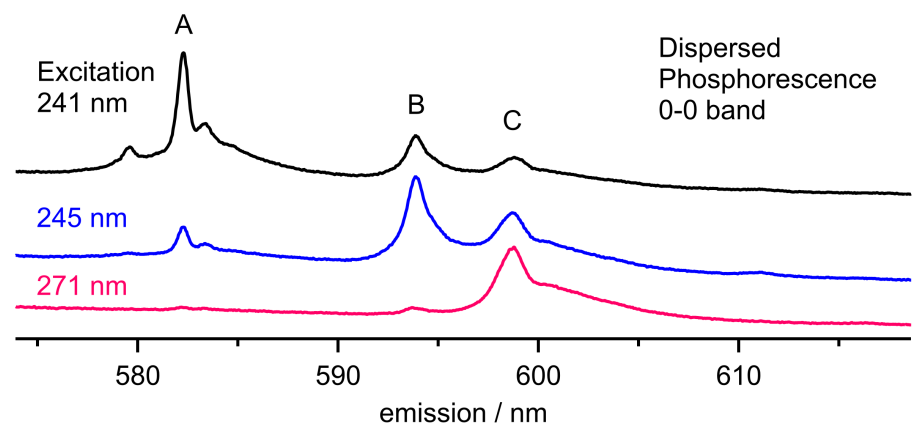

$\mathrm{HC}_{9} \mathrm{~N}$

Phosphorescence in Acetonitrile (Solid@20 K)
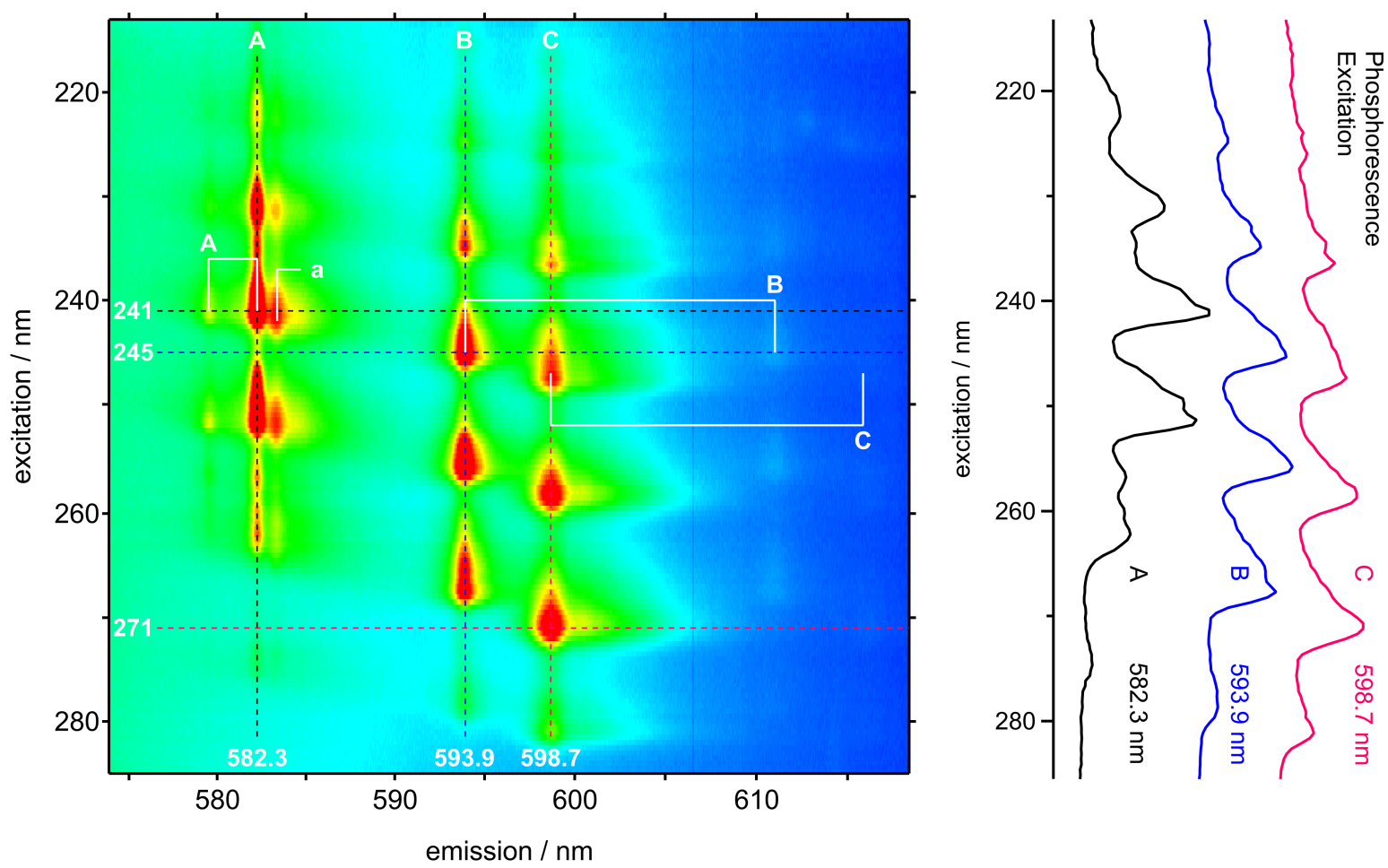


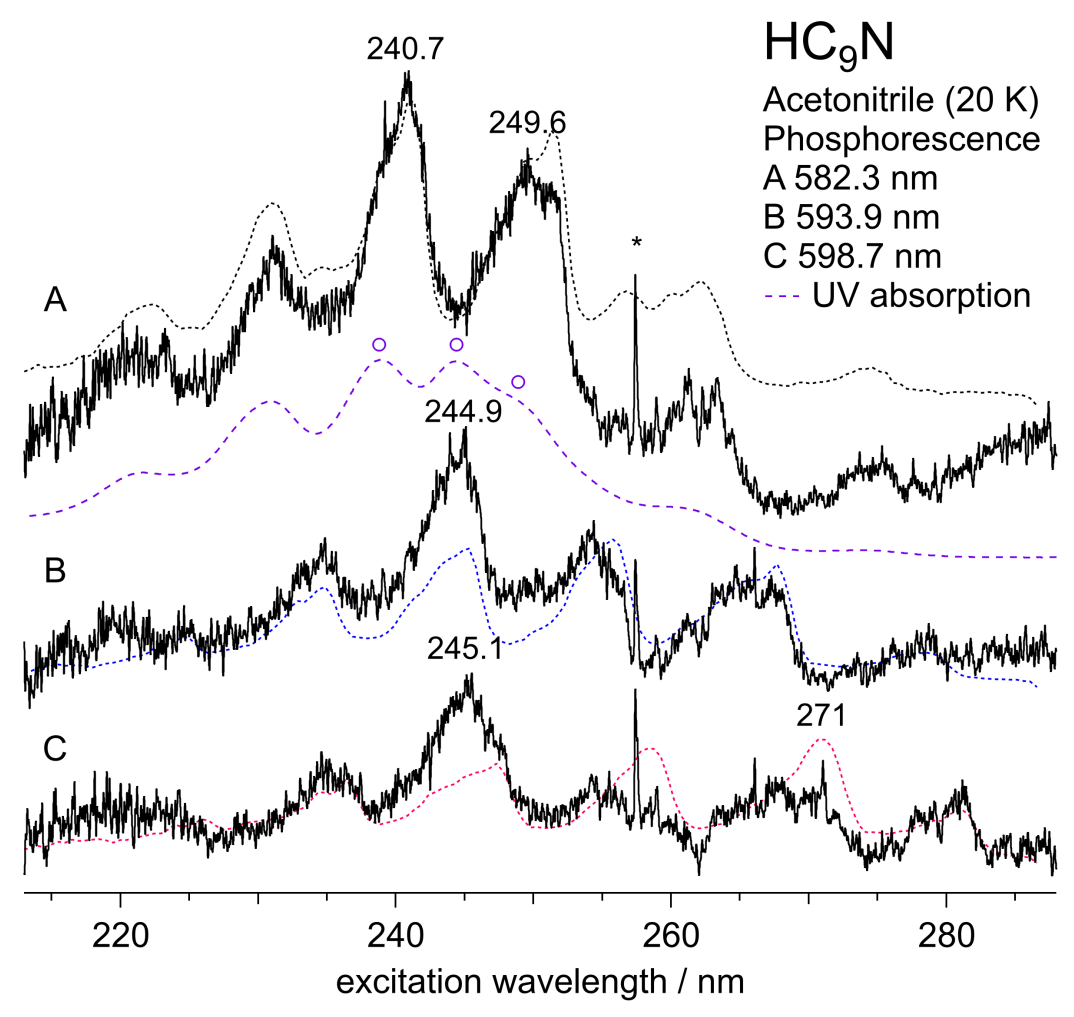



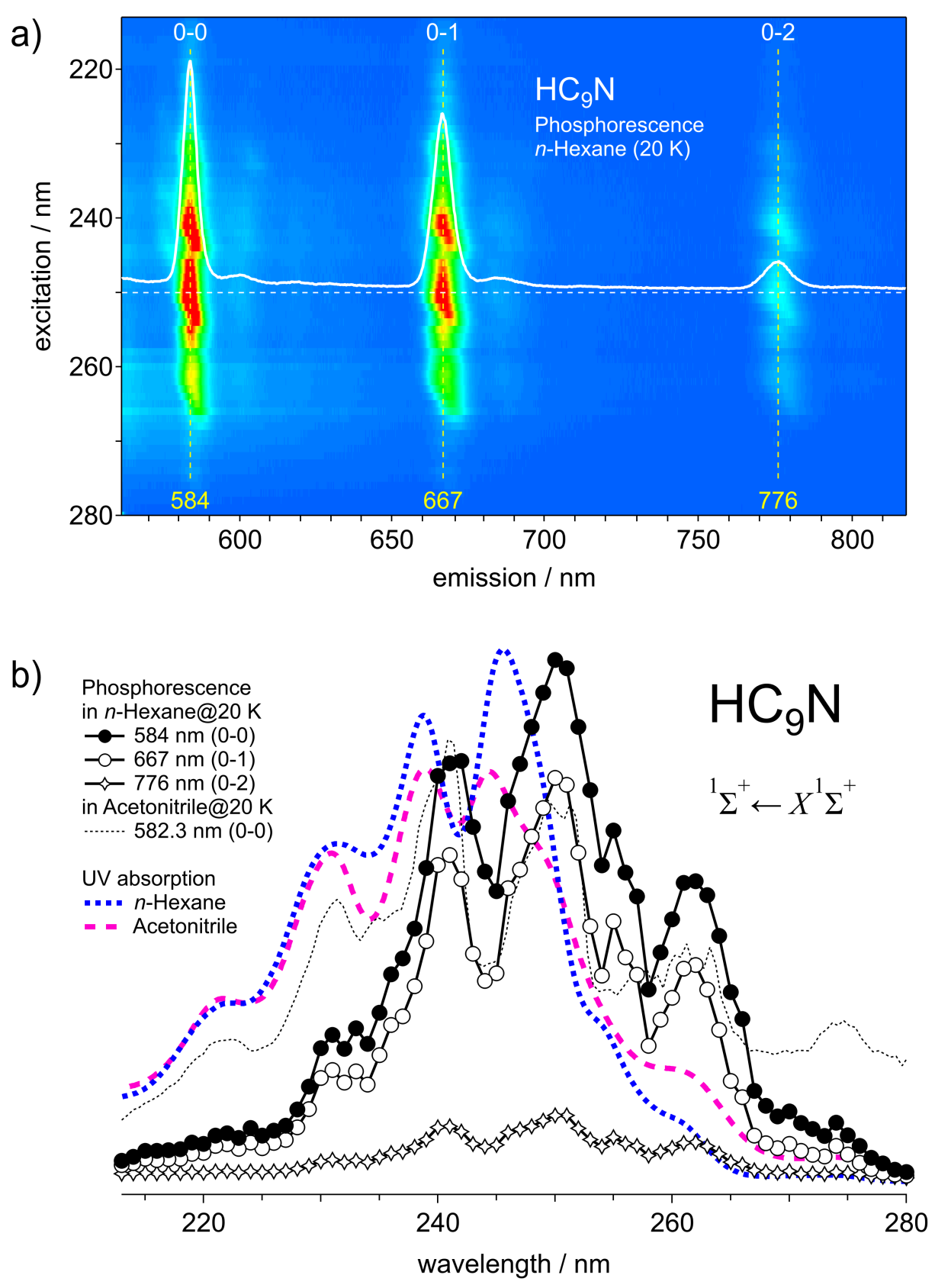


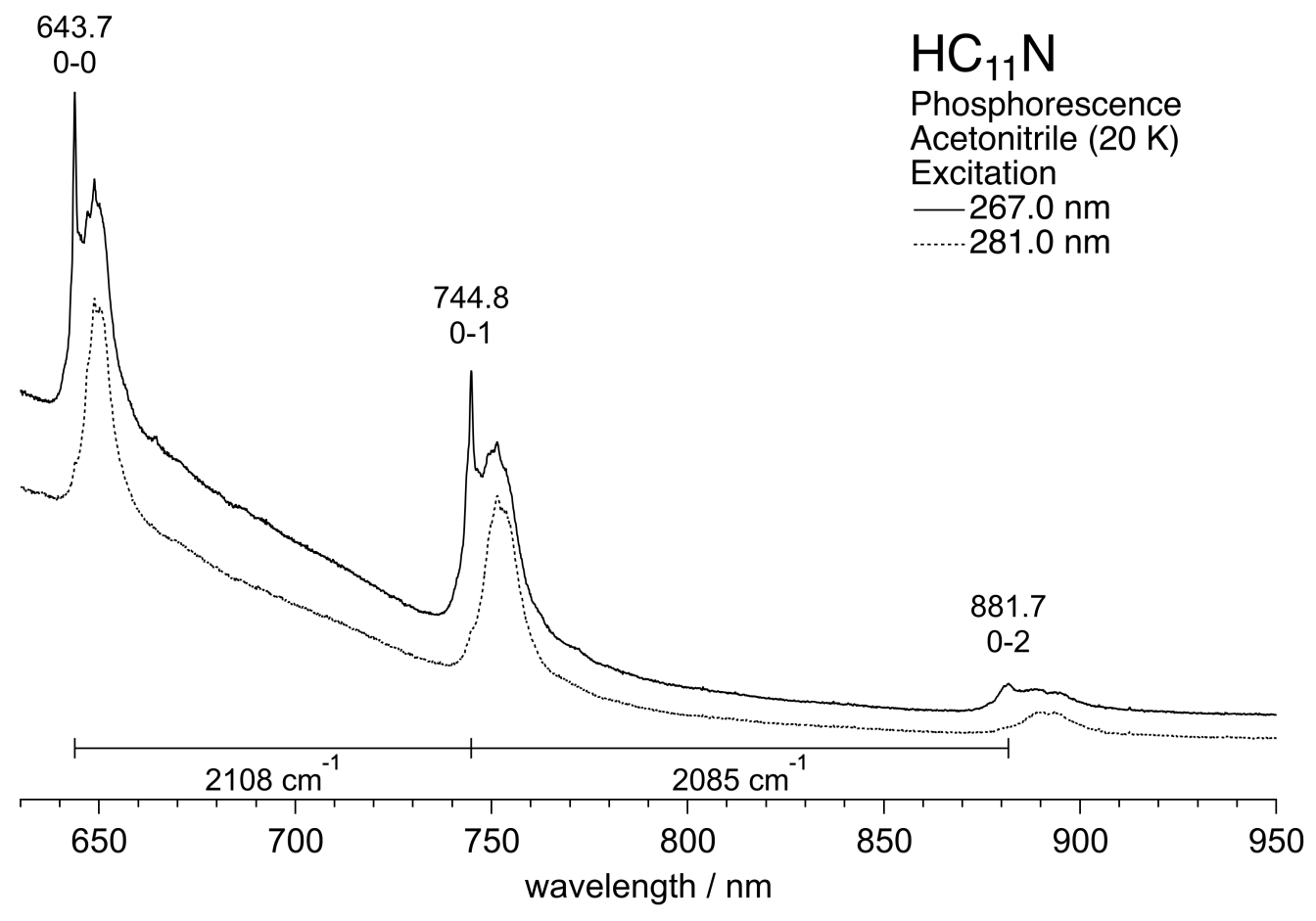




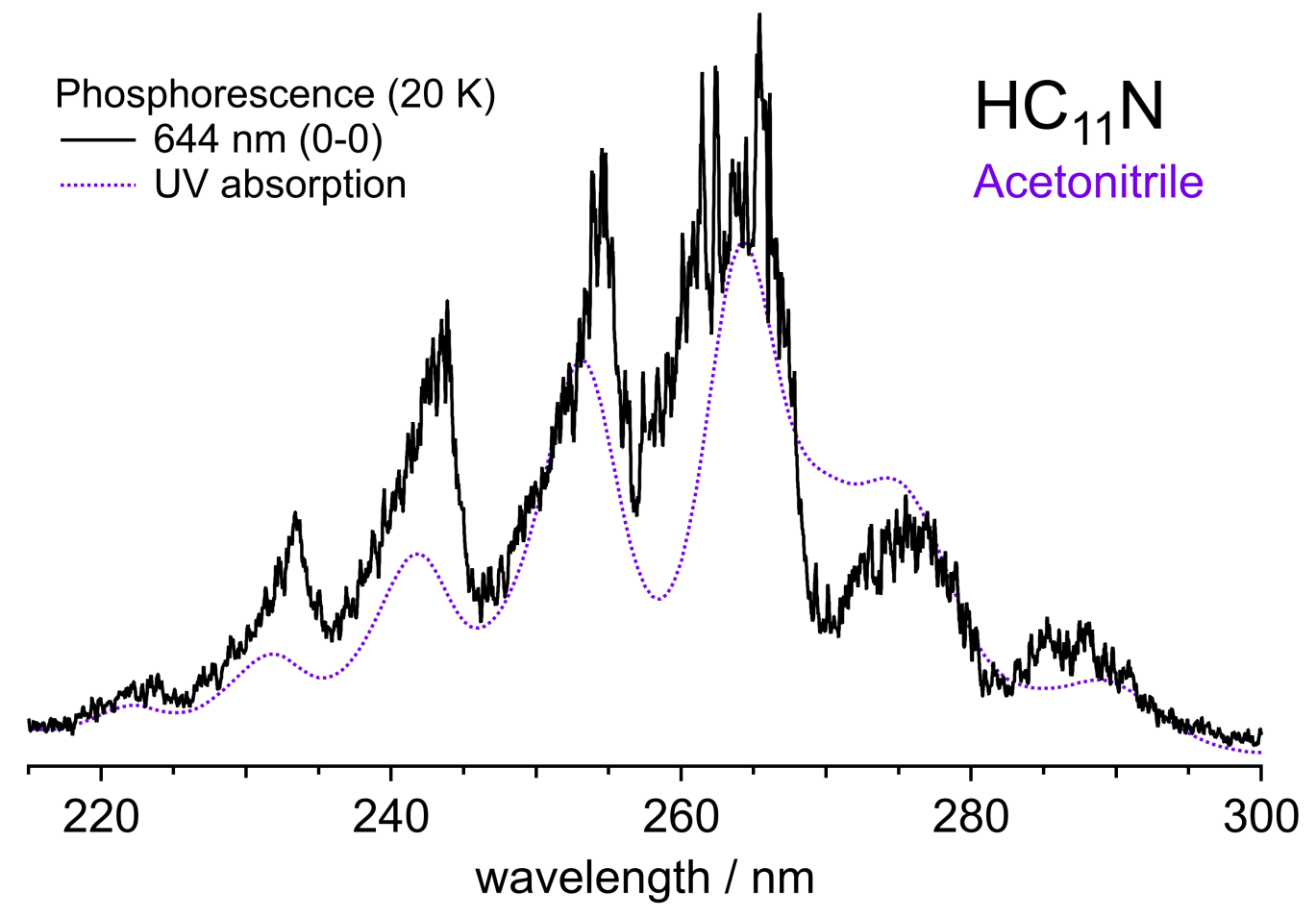



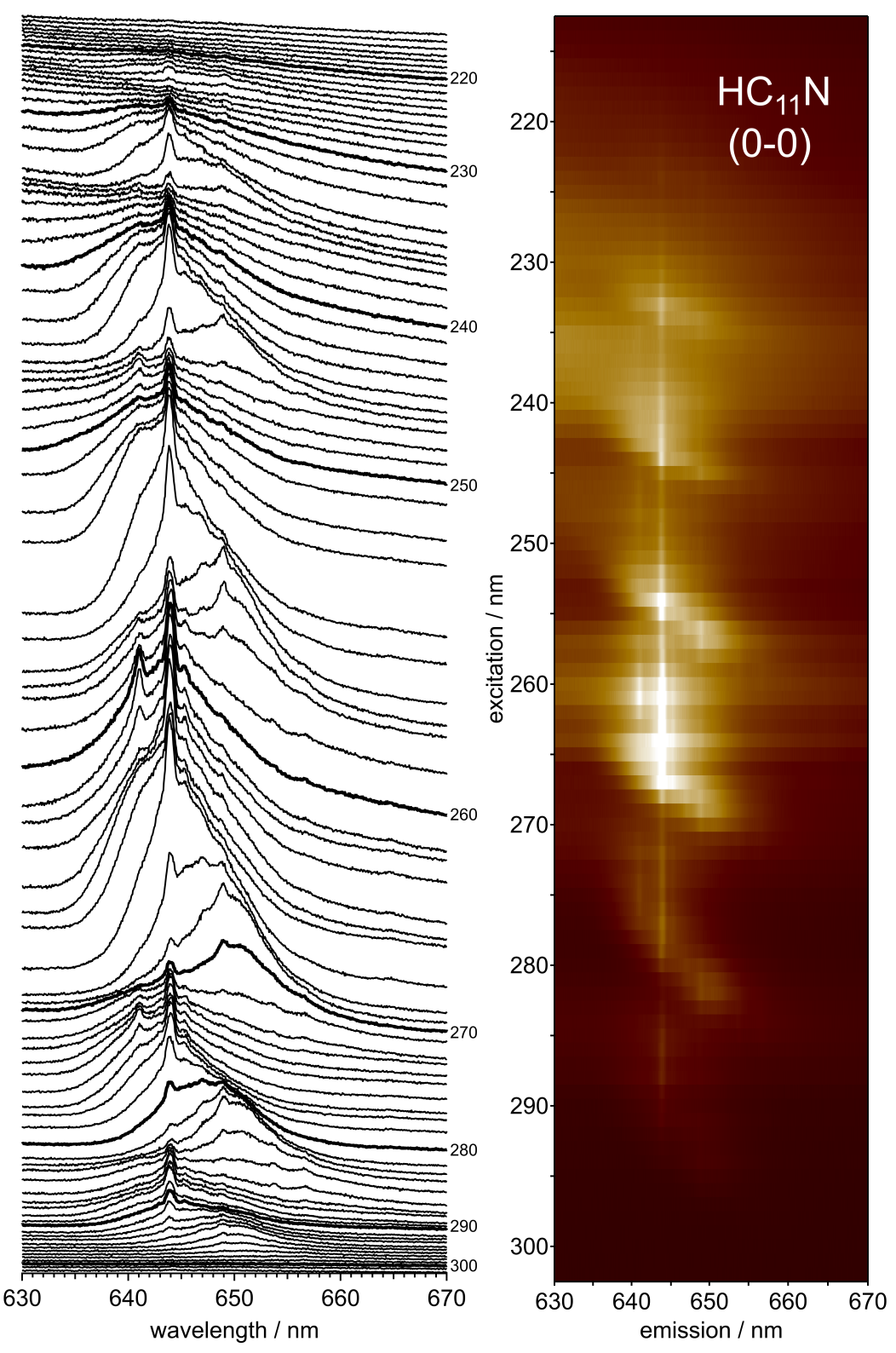


\section{Highlights}

Phosphorescence of cyanopolyyne molecules of $\mathrm{HC}_{9} \mathrm{~N}$ and $\mathrm{HC}_{11} \mathrm{~N}$ at $20 \mathrm{~K}$

$>$ Resonance Raman spectroscopy and IR spectra of $\mathrm{HC}_{9} \mathrm{~N}$ and $\mathrm{HC}_{11} \mathrm{~N}$

$>$ Solid acetonitrile or $n$-hexane matrix hosts provides multiple trapping sites 


\section{Declaration of interests}

$\bigotimes$ The authors declare that they have no known competing financial interests or personal relationships that could have appeared to influence the work reported in this paper.

$\square$ The authors declare the following financial interests/personal relationships which may be considered as potential competing interests: 